July 131994 Price: \$68. (text full color map w/topo)

$\$ 20$. (text, full color map w/ section grid

(no topo])

\$56. (text, mylar map in color w/section grid [oo topo])

Division of Geological \& Geophysical Surveys

PUBLIC-DATA FILE 94-39

\title{
PROGRESS REPORT ON THE GEOLOGY AND MINERAL RESOURCES OF THE NOME MINING DISTRICT
}

by

T.K. Bundtzen, R.D. Reger, G.M. Laird, D.S. Pinney, K.H. Clautice, S.A. Liss, and G.R. Cruse

July 1994

THIS REPORT HAS NOT BEEN REVIEWED FOR TECHNTCAL CONTENT (EXCEPT AS NOTED IN TEXT) OR FOR CONFORMITY TO THE EDITORIAL STANDARDS OF DGGS.

THIS DOCUMENT SHOULD NOT BE CITED EXCBPT AS

"WRITTEN COMMUNICATION, 1994."

Released by

STATEOFALASKA

DEPARTMENT OFNATURALRESOURCES

Division of Geological \& Geophysical Surveys

794 University Avenue, Suite 200

Fairbanks, Alaska 99709-3645 


\section{CONTENTS}

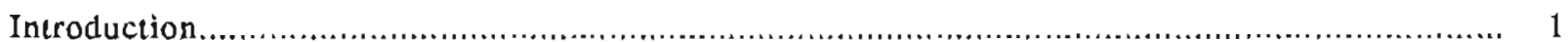

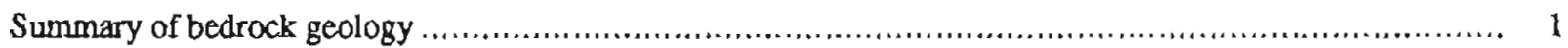

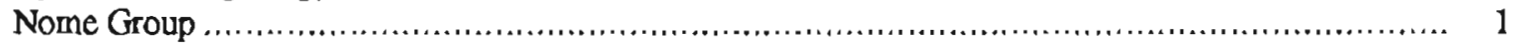

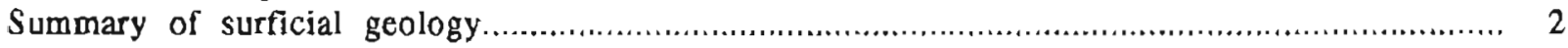

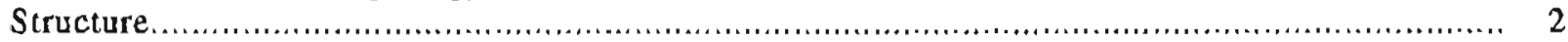

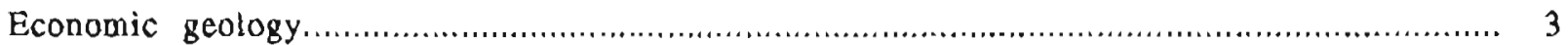

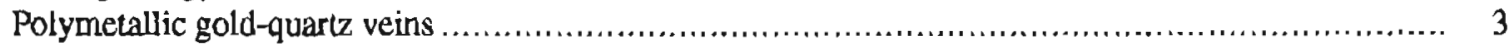

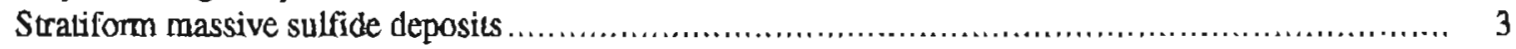

Carbonate hosted stratabound polymetallic deposits...................................................... 4

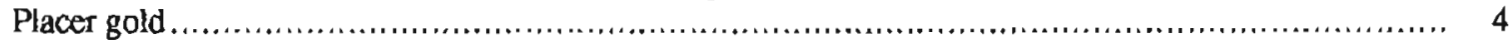

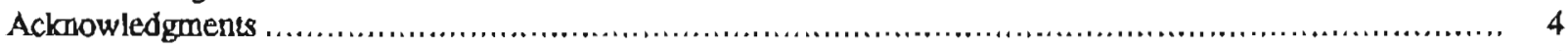

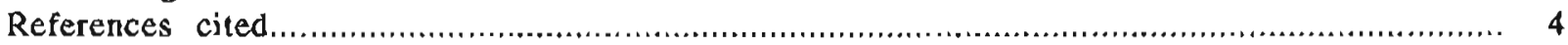

\section{TABLES}

Table 1. Major oxide analyses and CIPW normative mineralogy from metamorphic rocks in the Nome Mining District, Seward Peninsula, Alaska....................................... 7

2. Sulfur isotopic ratios from sulfide and sulfate specimens from mineral deposits in the Nome Mining Districh Seward Peninsula, Alaska............................................ 8

3. Geochemical determmations from selected mineral deposits, occurrences, and bedrock types, Nome mining districh Alaska ............................................................ 9

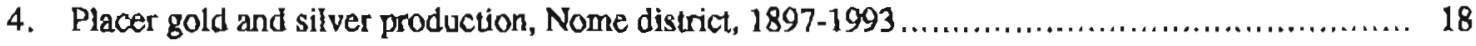

\section{SHEETS}

Sheet 1 Preliminary geologic map of Nome mining district

2 Sample location map showing trace element, major oxide, and sulfur isotope sample sites, Nome mining districk, Alaska 


\title{
PROGRESS REPORT ON THE GEOLOGY AND MINERAL RESOURCES OF THE NOME MINING DISTRICT
}

\author{
by \\ T.K. Bundtzen, R.D. Reger, G.M. Laird, D.S. Pinney, \\ K.H. Clautice, S.L. Liss, and G.R. Cruse
}

\section{INTRODUCTION}

During July 1993, the Alaska Divlsion of Geological and Geophystcal Surveys conducted bedrock and surficial geologic mapping of about $1,900 \mathrm{~km}^{2}\left(590 \mathrm{mi}^{2}\right)$ in the Nome mining district of western Alaska. This project was undertaken as a result of a Capital Improvements Project appropriation passed by the 1993 Alaska Legislature to begin detailed geological and mineral resource investigations of Alaskan mining districts using atrborne, multispectral geophysical surveys and 1;63,360-scale geological mapping. We emphasize that the results presented here are preliminary data acquired during 1993-1994. More field work will be completed during the summer of 1994, and a complete summary of the Nome district studies will be released pending completion of field, office, and laboratory investigations.

This preliminary report consists of: (1) the geologic map accompanied by complete map unit descriptions (sheet 1); (2) data tables that depict major oxide, sulfur isotope, and trace element analyses, and annual gold production statistics (tables 1-4); and (3) a brief summary text.

\section{SUMMARY OF BEDROCK GEOLOGY}

Twenty-two metasedimentary, metavolcanic, and metaplutonic bedrock units were delineated during our investigations. All rock units have been regionally metamorphosed. Throughout the following discussion, the reader is referred to data presented on the geologic map (sheet 1).

\section{NOME GROUP}

The regionally metamorphosed rocks of the Nome mining district were first described by Brooks and others (1901), whose work was supplanted by more detailed mapping and petrographic studies by Collier (1902), Knopf (1908), Smith (1910), and Moffit (1913). The high-grade regionally metamorphosed rocks of the central Kigluaik Mountains were referred to by Moffit (1913) as the Kigluaik Group; he defined the lower rank, regionally metamorphosed units in the Nome mining district as the Nome Group. Moffit (1913) and Smith (1910) flrst identified glaucophane in the Nome Group, which is now regarded as one of the largest, high P-low T, blueschistfacies metamorphic terranes in the world. Many modern workers including Sainsbury and others (1970), Forbes and others (1984), Thurston (1985), Evans and Palrick (1989), Till (1980), Sturnick (1984), Patrick and Evans (1989), Miller and Hudson (1991), and Miller and others (1992) have published detailed petrographic and radiometric age dating studies of metamorphic rocks in and near the study area. Nokleberg and otbers (1993) include the Nome Group into the Seward Terrane, an offset contineatal margin ranging in age from PreCambrian(?) to Late Paleozoic.

All regionally metamorphosed rocks in the study area bave been assigned to the Nome Group, although some small metamorphosed intrusions may be unrelated to the other units. We bave benefited from geologic map studies completed by the previously mentioned early workers and by Herreid (1970) and Hummel (1962a,b). Till and otbers (1986) and Till and Dumoulin (in press) believe that the Nome Group constitutes a coherent lithostratigraphic succession, which consists of four units: (1) a basal, complexly deformed quartz-rich pelitic schist, which may be correlative with the Solomon Schist of Smith (1910) and Moffit (1913); (2) a "mixed unit" of mafic and pelitic schists and marble; (3) a mafic-dominated schist package, which may be correlative with the Casadepaga Schtst of Smith (1910); and (4) an "impure marble unit". We have tentatively subdivided the Nome Group rocks of the study area into the first three units of Till and others (1986), which, in the study area, are locally repeated by thrust faults.

Smith (1910) and Moffit (1913) believed the Nome Group is Paleozoic in age. However, Sainsbury and others (1970) regarded all Nome Group lithologies as Precambrian in age, on the basis of inferred stratigraphic position and 
limited Rb-Sr isotopic-age determinations. Till and others (1986) have reported Ordovician to Devonian conodonts from marble units in the Nome Group. We assign a Precambrian-Paleozoic age to all Nome Group rocks mapped in the study area pending results of U-Pb isotopic-age dating of felsic metavolcanic rocks in the mixed unit and further geological mapping to the east of the study area in 1994.

\section{SUMMARY OF SURFICIAL GEOLOGY}

Most of the area is covered by drift laid down during past glaciations. Drift of the oldest advance, the Sinuk glaciation of early Pleistocene(?) age, is exposed in the vicinity of Cape Nome in the southeastem comer of the map area. The most extensive surface drift sheet was deposited during the Nome River glactation of middle Pleistocene age. Ice of this advance flowed southward out of cirques in the Kigluaik Mountains and coalesced with local ice from cirques and small ice caps in the uplands north of Nome. Ice of the Nome River glaciation filled the major trunk streams near Nome and extended southward several kilometers into present day Norton Sound (Hopkins and others, 1960; Nelson and Hopkins, 1972, Kaufman and Hopkins, 1989; Kaufman and others, 1991).

The subsequent Stewart River Glaciation spread out of the Kigluaik Mountains as far as $10 \mathrm{~km}$ beyond the mountain front. Ice from Kigluaik Mountain sources joined with local ice from small, north-facing cirques in the uplands immediately south of Kigluaik Mountains. The Stewart River glaciation is thought to be older than the last major interglaciation (Kaufman and Hopkins, 1986; Kaufman and others, 1988).

Slightly modified drift of the Salmon Lake glaciation forms lobate moraines that nearly reach as far south as the Stewart River terminal moraines (Kaufman and Hopkins, 1989). Radiocarbon dating indicates that this ice expansion occurred more than 33,000 years ago (Kaufman and others. 1989).

During and after these ice expansions, fluvial, colluvial, and marine processes modified the landscape, formed gravel-rich alluvial valley fills, and beach and terrace sediments. Fine-grained estuarine deposits were laid down in lower streams close to the present coast of Norton Sound.

\section{STRUCTURE}

All bedrock geologic units have been subjected to blueschist-facies metamorphism, which in turn was retrograded to T-P conditions of the greenschist facies. In the study area, earlier high P-low $T$ mineral assemblages are frequently altered due to the strong, retrogressive greenschist metamorphism. Earlier metamorphic minerals such as garmet, chloritoid, glaucopbane, hornblende, and calcic plagioclase are frequently rolled in a granoblastic fabric. Axial-plane schistosity parallel to compositional banding is the dominant "S 1 " surface measured in outcrop; later "S 2 "cleavage or foliztion is transposed at low angles in a westward vergence across lithologic or compositional banding, indicating that low-angle thrusting or recumbent folds have deformed the Nome Group in the study area. Stretched mineral lineations, isoclinal fold plunges, and mica crenulations (sheet 1) record deformation that postdates or ginal schistosity developed during the prograde, blueschist-facies event.

Lithostravigraphic units of the mixed unit are repeated by probable late synmetamorphic thrust faults south of Aurora Creek and east of Mount Distin. In the former area, metaturbidite schist (pCPzt), felsic metavolcanic schist (pCPzsf), black quartzite (pCPzsg), and "lumpy", porphyroclastic schist (pCPzpm) exposed in the Aurora Creek area are repeated in the Hungary Creek drainage implying a low-angle throw of approximately $7 \mathrm{~km}$. In the Basin Creek drainage, albite mafic schist and metabasite of the Casadepaga Schist(?) are thrust over the upper portion of the mixed unit. Other thrust faults are mapped on the basis of sheared contacts, low-angle mylonite zones, and outcropscale discontinuities, but the significance of offset along these faults is unknown.

Large-scale, younger structures include the north-trending Twin Mountain anticline, and nortbeast-trending high-angle faults. Pelitic, felsic, mafic, and carbonate-dominated lithologies of the mixed unit at Aurora Creek on the west are repeated at Dexter Mountain and Goldbotom Creek on the east by the Twin Mountain anticline, which largely defines the distribution of Nome Group lithologies in the study area.

The well known Anvil Creek fault trends north 40 to 55 degrees east up Anvil Creek (sheet 1), and juxtaposes metaturbidite schists on the west with pelitic, graphitic, metavolcanic, and calcareous lithologies on the east. A significant metafelsite center and interbedded pelitic schist and marble on Dexter Creek are equivalent to felstc metavolcanic schists and exhalite at Aurora Creek. The lithostratigraphic correlation indicales that the amount of postulated vertical movement along the Arvid fault is about $1.5 \mathrm{~km}$.

The Penny River fault (after Herreid, 1970) right laterally offsets the metavolcanic schist section at Aurora Creck aboult $4 \mathrm{~km}$ from an equivalent section on the east side of Penny River (sheet 1). 
Other significant northeast-trending high-angle faults include the Rodine fault (after Hawley and Buxton, oral comm. 1993), and the Oregon Creek-Charley Creek fault, both of which show right-lateral throws ranging from 1 to $3 \mathrm{~km}$.

Smaller less well defined, northwest-rending high-angle faults such as the Boulder Creek fault also cut the metamorphic rocks throughout the map area, and are probably more extensive than shown on the geologic map (sheet 1).

\section{ECONOMIC GEOLOGY}

Four types of metallic mineral resources have thus far been identified in the study area: (1) gold-polymetallicquartz-carbonate veins cutting pelitic and mafic scbists and carbonates, belleved to have formed as a result of regional metamorphism; (2) strat form, massive sulfide-barite deposits associated with felsic metavolcanic schist and metafelsite centers; (3) massive sulfide-iron deposits hosted in carbonate-dominated terranes and of uncertain origin; and (4) heavy mineral placer deposits, that have accounted for almost all of the metallic mineral production in the sturdy area.

All of our investigations of mineral resources are still in progress, and the results presented here are preliminary.

\section{Polymetallic Gold Quartz Veins}

Polymetallic-gold-quartz-carbonate mineral deposits contain most of the significant lode gold resources currently known in the Nome district. One depasit at Rock Creek (sheet 1) bas been recently evaluated by the mineral industry and contains a drill-inferred reserve amounting to 6.1 million tonnes grading $2.4 \mathrm{~g} /$ tonnc gold or 14.6 tonnes $(475,200$ ounces) of contained gold (Bundzen and others, 1992). The polymetallic gold quartz deposits contain arsenic, antimony, silver, zinc, lead, copper and tungsten in addition to the gold (table 3). Other important deposits include gold-tungsten deposits at Sophie Gulch, the gold-antimony deposits in the Mt. Distin area, the goldbismuth-arsenic vein deposits of the Charley Creek area, and the Rodine and other recently discovered deposits in the "Gold Hill trend" west of Snake River. At least three vein types are recognized: (1) early chalcopyrite-sphalertequartz-carbonate veins that appear as boudins rolled around Fl fold axes; (2) saddle reef quartz-gold-polysulfide veins (example; Rodine and McDuffy Prospects) that crosscut host schists at low angles to schistosity; and (3) britle vein systems that clearly crosscut metamorphic stratigraphy at high angles (example; Rock Creek; Sliscovich). All could be interpreted to have formed during various stages of dewatering of a metamorphic pile during Barrovian style greenschist-facies metamorphism and associated plutonism, as suggested for the origins of metallic vein mineralization at Rock Creek, (Apodaca, 1992) and Mt. Distin and Bluff (Ford, 1990).

Many of the gold-bearing vein deposits in the Nome district are hosted in metamorphosed clastic rocks interpreted to be turbidites and related deepwater sediments. The Rock Creek, Mt. Distin, and Gold Hill mineralized areas are similar to other turbidite hosted gold regions worldwide such as the saddle reef systems of the Meguma Terrane of Nova Scotia, Canada (Haynes, 1986; Sangster, 1990), the gold-scheelite vein deposits in the Otago and Reefton districts in New Zealand (Paterson, 1982; Lee and others, 1989; Henley and others, 1976; Lew and Corner, 1988), or the gold vein deposits hosted in the Valdez Group of southern Alaska (Goldfarb and others, 1986).

Limited sulfur isotopic analyses conducted during our investigations show light del-34 values ranging from -1.6 to +3.6 and averaging about +0.2 from sulfides in six gold-polymetallic vein deposits of the study area (able 2).

\section{Stratiform Massive Sulfide Deposits}

Stratiform massive sulfide-barite deposits in the Aurora Creek area and at the Christopherson Prospect were first described by Herreld $(1966,1970)$, and evaluated by the mineral industry in the mid-1970s and more recently in the 1990s. During our brief investigations we mapped a complex package of muscovite-feldspar metavolcanic schist, carbonate, pelitic schist, and graphitic scbist informally referred to as the "Aurora Creek section" (pCPzf, pCPzsf, pCPzsg; sheet 1). Lenses and disseminations of sphalerite, galena, chalcopyrite, and massive barite appear to be parallel to original compositional banding of host metavolcanic and carbonaceous schist for over $4 \mathrm{~km}$ of strike length. Mineralization is complexly folded along with host rocks indicatinging a premetamorphic origin for the mineralized zones. Zones of intense tourmalinization are present in wallrock adjacent to the massive sulfide-barite 
deposits. Limited sulfur isotopic analyses yield heavy del-34 values ranging from +5.5 to +26.3 , typical of seawater contaminated sulfur in submarine volcanogenic massive sulfide settings (table 2). Selected samples from the Aurora Creek deposits coneain up to $15.86 \%$ zinc, $1.38 \%$ lead, 659 porn copper, and anomalous cadmium, barium, silver and gold (table 3).

Tourmaline enrichment in felsic schist horizons could indicate hydrothermal activity related to premetamorphic submarine volcanism in other portions of the study area.

\section{Carbonate-hosted Stratabound Polymetallic Deposits}

Carbonate-hosted sulfide-barite-fluorite-iron deposits have been described from the Sinuk River drainage in fair detail by Mulligan and Hess (1965) and Herreid (1970). The origin of these sulfide-bearing gossans is uncertain. Mineral deposit types proposed for these polymetallic occurrences include sedex, volcanogenic massive sulfide, intrusive related replacement, and hydrothermal vein. One sulfur isotopic analysis collected from the Quarry Prospect yielded a del-34 value of +10.8 from galena (sheet 1 ; table 2), which contrasts with light del-34 sulfur values found in polymetallic veins in the study area.

\section{Placer Gold}

Placer gold has been the principal metallic mineral resource mined in the study area. From 1898 to 1993, an estimated 4,822,569 ounces (150 metric tonnes) gold have been produced from strearn, hillslope or colluvial, glacial, and marine strandline placer deposits throughout the study area (table 4), making the Nome district Alaska's second largest producer of placer gold. The most significant placer deposits are concentrated along modern and ancestral strandline deposits on the Coastal Plain, in streams draining Anvil Mountain, and in tributaries of the Nome, Osborm, and Snake Rivers (Collier and others, 1908 and Cobb, 1974). An estimated 70 percent of the gold has been produced from the strandline deposits, but rich paystreaks bave been mined from glacial, colluvial, and stream deposits.

Glacial scouring milled gold lodes and dispersed much of the gold that eventually formed placer deposits in the Nome mining districL During and after several ice expansions, fuvial, colluvial, and marine processes concentrated gold to form economic placer deposits. One byproduct of the long and extensive placer gold mining activities has been the production of large volumes of high-quality, washed tailings suitable for foundation materials for highways and settlement areas.

Placer geology research is in progress.

\section{ACKNOWLEDGMENTS}

We thank Chuck Hawley and Ms. Cindy Buxton of Kennecott Exploration Inc. for sharing their extensive knowledge of the geology of the Gold Hill and Aurora Creek areas. Allison Till (U.S. Geological Survey) spent two days with us during the 1993 field season, and demonstrated a defendable, metamorphic lithostratigraphy for the Nome Group in the western Solomon Quadrangle. Much appreciation is extended to Tom Sparks of Bering Straits Native Corporation and Irene Anderson of Sitnausuk Corporation, who helped secure and provide for logistical services during our studies. Joe Fisher kindly allowed access to Alaska Gold Company lands in the Nome district.

\section{REFERENCES CITED}

Apodaca, L.E, 1992, Fluid inclusion study of the Rock Creek area, Nome Mining District, Alaska: in, Bradley, D.C., and Dusel-Bacon, Cynthia, eds., Geologic studies in Alaska by the U.S. Geological Survey, 1991: U.S. Geological Survey Bulletin 2041, p.3-12.

Brooks, A.H., Richardson, G.B., Collier, A.J., and Mendenhall, W.C., 1901, Reconnaissances in the Cape Nome and Norton Bay Regions, Alaska. in 1900: U.S. Geological Survey Special Publication, 185 p.

Bundtzen, T.K., Swainbank, R.C., Wood, J.E., and Clough, A.H., 1992, Alaska's Mineral Industry-1991: Alaska Division of Geological \& Geophysical Surveys Spectal Report 46, 89 p.

Cobb. E.H., 1974, Placer deposits of Alaska, U.S. Geological Survey Bulletin 1374, 217 p.

Collier, A.J., 1902, A reconnaissance of the northwestem portion of the Seward Peninsula, Alaska: U.S. Geological Survey Professional Paper 2, 70 p. 
Collier, A.J., Hess, F.L., Smith, P.S., and Brooks, A.H., 1908, The gold placers of parts of Seward Peninsula, Alaska, including Nome, Council, Kougarok, Por Clarence, and Goodhope Districts: U.S. Geological Survey Bulletin 328, $343 \mathrm{p}$.

Evans, B.W., and Patrick, B.E., 1989, Pbengite 3-T in high pressure orthogneisses, Seward Peninsula, Alaska: Canadian Mineralogist, vol. 25, p. 141-158.

Forbes, R.B., Hamilton, Tom, Tailleur, L.L., Miller, T.P., and Patton, W.W., Jr., 1984, Regional progressive high pressure metamorphism, Seward Peninsula , Alaska: Journal of Metamorphic Geology, vol. 2, no 1, p.43-54.

Ford, R.C., 1990, Geology, geochemistry, and age of gold lodes at Bluff, and Mt. Distin, Seward Peninsula, Alaska: unpublished Ph.D. dissertation, Colorado School of Mines, Colorado, $302 \mathrm{p}$.

Goldfarb، R.J., Leach, D.L., Miller, M.L., and Pickthorn, W.J., 1986, Geology, metamorphic setting, and genetic constraints of epigenetic lode-gold mineralization with the Cretaceous Valdez Group, south-central Alaska: in, Turbidite bosted gold deposils, Geological Association of Canada Special Paper 32, p.87-105.

Haynes, S.J., 1986, Grology and chemistry of turbidite-hosted gold deposits, greenschist facies, eastern Nova Scotia, Canada: in Turbidite Hosted Gold Deposits, Geological Association of Canada Special Paper 32, p.161-177.

Henley, R.W., Norris, R.J., and Patterson, CJ, 1976. Multistage ore genesis in the New Zealand geosyncline, a history of post-metamorphic lode emplacement: Mineral Deposita, vol. 11, p. 180-196.

Herreid, Gordon, 1966. Pretiminary geology and geochemistry of the Sinuk River area, Seward Peninsula, Alaska: Alaska Division of Geological \& Geophysical Surveys Geologic Report 24, 19 pages.

1970, Geology and geochemistry of the Sinuk River area, Seward Peninsula, Alaska: Alaska Division Geological \& Geophysical Surveys Geologic Repor 36, 63 p.

Hopkins, D.M., MacNeil, F.S., and Leopold, E.B., 1960, The coastal plain at Nome, Alaska: A late Cenozoic type section for the Bering Strait region, in Sorgenfrei, Theodor, ed., Report of the Twenty-first Session, International Geological Congress, Part 4, 343 p.

Hummel, C.L., 1962a, Preliminary geology of the Nome C-1 Quadrangle, Seward Peninsula, Alaska: U.S. Geological Survey Map MF-247., 1 sheet, scale 1:63,360.

1962b, Preliminary geology of the Nome D-1 Quadrangle, Seward Peninsula, Alaska: U.S. Geological Survey Map MF 248, 1 sheet, scale 1:63,360.

Kaufman, D.S., Calkin, P.E., Whitford, W.B., Przybyl, B.J., Hopkins, D.M., Peck, B.J., and Nelson, R.E., 1989, Surficial geologic map of the Kigluaik Mountains, Seward Peninsula, Alaska: U.S. Geological Survey Miscellaneous Field Studies Map MF-2074, 1 sheel, scale 1:63,360.

Kaufman, D.S., and Hopkins, D.M., 1986, Glacial history of the Seward Peninsula, in Hamilton, T.D., Reed, K.M., and Thorson, R.M., eds., Glaciation in Alaska--the geologic record: Anchorage, Alaska Geological Society, p. $51-78$.

1989, Late Cenozoic geologic controls on placer-gold distribution in the Nome nearshore area, in Dover, H.H., and Galloway, J.P., ed's., Geologic studies in Alaska by the U.S. Geological Survey, 1988: U.S. Geological Survey Bulletin 1903, p. 26-45.

Kaufman, D.S., Hopkins, D.M., and Calkin, P.E., 1988, Glacial geologic history of the Salmon Lake area, Seward Peninsula, in Galloway, J.P., and Hamilton, T.D., eds., Geologic studies in Alaska by the U.S. Geological Survey during 1987: U.S. Geological Survey Circular 1016, p. 91-94.

Kaufman, D.S., Walter, R.C., Brigham-Gretre, Julie, and Hopkins, D.M., 1991, Middle Pleistocene age of the Nome River Glaciation, northwestern Alaska: Quatemary Research, vol. 36, no. 3, p. 277-293.

Knopf, Adolph, 1908, Geology of the Seward Peninsula tin deposits, Alaska: U.S. Geological Survey Bulletin 358, $71 \mathrm{p}$.

Moffit, F.H., 1913, Geology of the Nome and Grand Central Quadrangles, Alaska: U.S. Geological Survey Bulletin $533,140 \mathrm{p}$.

Mulligan, J.J., and Hess, H.D., 1965, Examination of the Sinuk Iron deposits, Seward Peninsula, Alaska: U.S. Bureau of Mines Open File Report 34 p.

Lee, M.C., Batt, W.D., and Robinson, P.C., 1989, The Round Hill gold-scheelite deposit, Macraes Flat, Otago, New Zealand: in, Kear, David, ed., Mineral Deposits of New Zealand-the Gordon J. Williams Memorial Volume, Monograph 13, p. 173-179.

Lew, J.H., and Comer, N.G., 1988, The Globe-Progress prospect, Reefton, New Zealand: Bicentennial Gold-1988, Melboume, Australia, p. 271-273.

Miller, E.L., Calvert, A.T., and Litlle, T.A., 1992, Strain-collapsed metamorphic isograds in a sillimanite gneiss dome, Seward Peninsula, Alaska: Geology, vol. 20, p.487-490. 
Miller, EL., and Hudson, T.L., 1991, Mid-Cretaceous extensional fragmentation of a Jurassic-Early Cretaceous compressional orogen: Tectonics, vol. 10, p. 781-796.

Nelson, C.H., and Hopkins, D.M., 1972, Sedimentary processes and distribution of particulate gold in the northern Bering Sea: U.S. Geological Survey Professional Paper 689, 27 p.

Nokleberg, W.J., Bundtzen, T.K., Grybeck, Donald, Koch, R.D., Eremin, R.A., Rozenblum, I.S., Sidorov, A.A., Byalobzhesky, G.S., Shpikennan, V.I, and Gorodinsky, M.E., 1993, Metallogenesis of mainland Alaska and the Russian Northeast: U.S. Geological Survey Open File Report 93-339, 5 sheets, scale 1:4,000,000, 291 p.

Paterson, C.J., 1982, Oxygen isotopic evidence for the origin and evolution of a scheelite ore-forming flutd, Glenorchy, New Zealand: Economic Geology, vol. 77, p. 1672-1687.

Patrick, BE., and Evans, B.W., 1989, Metamorphic evolution of the Seward Peninsula blueschist terrane, Alaska: Journal of Petrology, vol. 30, p. 531-556.

Sainsbury, C.L., Coleman, R.G., and Kachadoorian, Reuben, 1970, Blueschist facies rocks of the Seward Peninsula. Alaska: U.S. Geological Survey Professional Paper 700-B, p. 33-42.

Sangster, A.L., 1990, Metallogeny of the Meguma Terrane, Nova Scotia: in Mineral Deposit Studies in Nova Scotia, Geological Survey of Canada Paper 90-8, p. 115-162.

Smith, P.S., 1910, Geology and milteral resources of the Solomon and Casadepaga Quadrangles, Seward Peninsula, Alaska: U.S. Geological Survey Bulletin 433, 234 p.

Sturnick, M.A., 1984, Regional metamorphism in the eastern Kigluaik Mountains, Seward Peninsula, Alaska: Geological Society of America Abstracts with Programs, vol. 16, p. 335.

Thurston, S.P., 1985, Structure, petrology, and metamorphic history of the Nome Group blueschists, Salmon Lake area, Seward Peninsula, Alaska: Geological Society of America Bulletin, vol. 96, p. 600-617.

Till, A.B., 1980, Crystalline rocks of the Kigluaik Mountains, Seward Peninsula, Alaska: unpublisbed M.S. Thesis, University of Washington, $97 \mathrm{p}$.

Till, A.B., Dumoulin, J.A.. Gamble, B.M., Kaufman, D.S., and Carroll, P.I., 1986, Preliminary geologic map and fossil data, Solomon, Bendeleben, and southern Kotzebue Quadrangles, Seward Peninsula, Alaska: U.S. Geological Survey Open File Report 86-276, 3 sheets, scale 1:250,000, 71 p.

Till, A.B., and Dumoulin, J.A., in press, Seward Peninsula: the Seward and York Terranes: in Plafker, George, and Berg H.C., eds., Geology of Alaska: Geological Society of America Decade of North American Geology, $23 \mathrm{p}$. 
Table 1. Major oxide analyses and CIPW normative mineralogy from selected metomorphic rocks, Nome mining district, Alaska

Major oxide analyses

\begin{tabular}{|c|c|c|c|c|c|c|c|c|c|c|c|c|c|c|c|}
\hline $\begin{array}{c}\text { Map } \\
\text { no. }\end{array}$ & $\begin{array}{c}\text { Sample } \\
\text { no. }\end{array}$ & $\begin{array}{c}\mathrm{SiO}_{2} \\
(\mathrm{wt} \%)\end{array}$ & $\begin{array}{l}\mathrm{TiO}_{2} \\
\text { (wt\%) }\end{array}$ & $\begin{array}{l}\mathrm{Al}_{2} \mathrm{O}_{3} \\
(\mathrm{w} \%)\end{array}$ & $\begin{array}{l}\mathrm{Fe}_{2} \mathrm{O}_{3} \\
\text { (wt \%) }\end{array}$ & $\begin{array}{c}\mathrm{FeO} \\
(\mathrm{wt} \%)\end{array}$ & $\begin{array}{c}\mathrm{MnO} \\
\left(w_{t} \%\right)\end{array}$ & $\begin{array}{c}\mathrm{MgO} \\
(\mathrm{w} \%)\end{array}$ & $\begin{array}{c}\mathrm{CaO} \\
(\mathrm{w} \%)\end{array}$ & $\begin{array}{l}\mathrm{Na}_{2} \mathrm{O} \\
(\text { w \%) }\end{array}$ & $\begin{array}{c}\mathrm{K}_{2} \mathrm{O} \\
\text { (wt \%) }\end{array}$ & $\begin{array}{c}\mathrm{P}_{2} \mathrm{O}_{5} \\
(\mathrm{wt} \%)\end{array}$ & $\begin{array}{l}\mathrm{Cr}_{2} \mathrm{O}_{3} \\
(\mathrm{wt} \%)\end{array}$ & $\begin{array}{c}\text { LOI } \\
(w \% \%)\end{array}$ & $\begin{array}{l}\text { Total } \\
\text { (w \%) }\end{array}$ \\
\hline 1 & $93 \mathrm{BT} 161 \mathrm{~A}$ & 68.83 & 1.06 & 14.82 & 2.11 & 3.64 & 0.04 & 1.30 & 0.33 & 0.07 & 3.20 & 0.18 & 0.03 & 2.46 & 98.07 \\
\hline 2 & $93 \mathrm{BT} 171$ & 75.39 & 0.30 & 13.31 & 0.59 & 1.03 & 0.01 & 0.72 & 0.25 & 7.02 & 0.05 & 0.09 & 0.01 & 0.66 & 99.43 \\
\hline 3 & $93 \mathrm{BT} 203$ & 46.93 & 2.07 & 14.53 & 4.28 & 10.55 & 0.22 & 6.36 & 7.55 & 2.55 & 0.05 & 0.14 & 0.02 & 3.23 & 98.48 \\
\hline 4 & $93 \mathrm{BT} 266$ & 54.86 & 1.08 & 17.14 & 2.39 & 5.85 & 0.03 & 3.67 & 1.23 & 7.20 & 0.05 & 0.09 & 0.02 & 4.94 & 98.55 \\
\hline 5 & $93 \mathrm{BT} 321$ & 56.20 & 1.04 & 18.28 & 5.45 & 3.27 & 0.07 & 3.86 & 1.06 & 3.19 & 3.11 & 0.19 & 0.02 & 3.91 & 99.61 \\
\hline 6 & 93GL2O4 & 66.13 & 0.99 & 12.36 & 3.93 & 2.06 & 0.10 & 2.87 & 3.36 & 2.91 & 1.01 & 0.16 & 0.01 & 3.36 & 99.25 \\
\hline 7 & 93 GL216 & 69.95 & 1.04 & 11.11 & 0.80 & 4.31 & 0.07 & 3.18 & 5.55 & 1.75 & 0.97 & 0.19 & 0.02 & 1.73 & 100.67 \\
\hline 8 & 93 GL236 & 67.79 & 0.83 & 13.66 & 1.40 & 4.12 & 0.05 & 2.37 & 1.43 & 3.16 & 1.66 & 0.09 & 0.02 & 2.45 & 99.03 \\
\hline 9 & $93 \mathrm{KCl} 104$ & 75.46 & 0.71 & 11.10 & 1.41 & 2.64 & 0.06 & 1.84 & 1.06 & 1.89 & 1.52 & 0.15 & 0.02 & 2.76 & 100.62 \\
\hline
\end{tabular}

CIPW normative minerals

\begin{tabular}{|c|c|c|c|c|c|c|c|c|c|c|c|c|c|}
\hline $\begin{array}{l}\text { Map } \\
\text { no. }\end{array}$ & $\begin{array}{c}\text { Sample } \\
\text { no. }\end{array}$ & Qtz & Cor & $\mathrm{O}_{\mathrm{r}}$ & $\mathrm{Ab}$ & An & $\mathrm{Di}$ & $\mathrm{Hy}$ & Mi & $\mathrm{Hm}$ & Ilm & Ap & Description \\
\hline 1 & 93BT161A & 54.508 & 11.706 & 19.993 & 0.626 & 0.489 & 0.000 & 6.875 & 3.235 & 0.000 & 2.129 & 0.441 & Feldspar muscovite schist (pCPzsf) \\
\hline 2 & $93 \mathrm{BT} 171$ & 33.002 & 1.487 & 0.299 & 60.143 & 0.661 & 0.000 & 2.754 & 0.866 & 0.000 & 0.577 & $0.2] 1$ & Metagranite (pCPzg) \\
\hline 3 & $93 \mathrm{BT} 203$ & 2.770 & 0.000 & 0.310 & 22.657 & 29.457 & 7.323 & 26.500 & 6.517 & 0.000 & 4.128 & 0.340 & Metabasite (pCPzb) \\
\hline 4 & $93 \mathrm{BT} 266$ & 1.854 & 3.441 & 0.316 & 65.092 & 5.892 & 0.000 & 17.288 & 3.703 & 0.000 & 2.192 & 0.223 & Metadiorite (Mzdg) \\
\hline 5 & $93 \mathrm{BT} 321$ & 19.081 & 8.563 & 19.207 & 28.210 & 4.200 & 0.000 & 10.047 & 7.968 & 0.201 & 2,064 & 0.460 & Albite schist (pCPzsm) \\
\hline 6 & $93 G L 204$ & 35.789 & 0.786 & 6.225 & 25.680 & 16.295 & 0.000 & 7.455 & 4.272 & 1.153 & 1.961 & 0.386 & Albite chlorite schist (pCPzsm) \\
\hline 7 & $93 G L 216$ & 39.254 & 0.000 & 5.794 & 1.969 & 19.808 & 5.541 & 11.020 & 1.173 & 0.000 & 1.997 & 0.445 & Metaturbidite schist (pCPztc) ${ }^{b}$ \\
\hline 8 & 93GL_236 & 35.604 & 4.433 & 10.159 & 27.690 & 6.738 & 0.000 & 11.426 & 2.102 & 0.000 & 1.633 & 0.216 & Feldspar muscovite schist (pCPzsf) \\
\hline 9 & $93 \mathrm{KC} 104$ & 54.033 & 4.883 & 9.180 & 16.345 & 4.374 & 0.000 & 7.363 & 2.090 & 0.000 & 1.378 & 0.355 & Graphitic quartzose schist (pCPrsg) $)^{\mathrm{b}}$ \\
\hline
\end{tabular}

analyses by Bondar-Clegg, Ldd, 130 Pemberton Ave, North Vancouver, B.C., Canada V7P2R5.

CIPW aomative data catculated with UAF/PETCAL program, University of Alaska, Fairbanks, Alaska 99709.

Sorns probably not applicable due to presumed metasedimentary parentage. 
Table 2. Sulfur isotopic ratios of sulfide and sulfate specimens from mineral deposits in the Nome mining district, Seward Peninsula, Alaska

\begin{tabular}{|c|c|c|c|c|c|}
\hline \multicolumn{6}{|c|}{ Sample } \\
\hline Charley Creek & $93 \mathrm{BT} 297 \mathrm{a}$ & Polymetallic-gold vein & Arsenopyrite & +0.6 & Hanging wall vein \\
\hline Charley Creek & $938 T 297 \mathrm{~b}$ & Polymetallic-gold vein & Arsenopyrite & +0.8 & Hanging wall vein \\
\hline Rock Creek & $908 T 302 a$ & Polymetallic-gold vein & Arsenopyrite & +0.9 & From bulk sample pit \\
\hline Rock Creek & $90 \mathrm{BT} 302 \mathrm{~b}$ & Polymetallic-goid vein & Galena & $-1,6$ & North of bulk sample pit \\
\hline Rock Creek & $90 \mathrm{BT} 302 \mathrm{c}$ & Polymetallic-gold vein & Galena & .0 .9 & North of bulk sample pit \\
\hline Unnamed & $93 \mathrm{BT} 177$ & Polymetallic-gold vein & Chalcopyrite & +0.3 & Early(?) F1 sulfude quartz vein \\
\hline Unnamed & $93 \mathrm{KCl} 16$ & Polymetallic-gold vein & Sphalerite & +3.6 & Early(?) Fl sulfide quartz vein \\
\hline Twin ML prospect & 93BT229a & Polymetalic-gold vein & Galena & -1.1 & Saddle reef system \\
\hline Twin ML prospect & 93BT229b & Polymetallic-gold vein & Arsenopysite & -0.6 & Saddle reef system \\
\hline Twin Mt. prospect & $93 \mathrm{~B} T 229 \mathrm{c}$ & Polymetallic-gold vein & Arsenopyrite & $-0,5$ & Saddle reef system \\
\hline Hed and Strand prospect & $93 \mathrm{BT} 335$ & Stibnite-sulfosalt vain & Lead-antimony sulfosalt & -0.2 & Vein in calc-schist near shaft \\
\hline Christopherson & 93BT197a & Stratiform sulfide & Barite & +26.3 & From ridge pit \\
\hline Christopherson & 93BT197b & Stratiform sulfide & Pyrite & +5.5 & From ridge pit \\
\hline Christopherson & $93 \mathrm{BT} 197 \mathrm{c}$ & Stratitorm sulfide & Gajena & +17.3 & From ridge pit \\
\hline Aurora Creek & $938 T 231$ & Stratiform sulfide & Sphalerite & +12.9 & From stream cut outcrops \\
\hline Quarry Prospect & 90BT337 & $\begin{array}{l}\text { Carbonate-hosted, stratiform } \\
\text { sulfide-barite }\end{array}$ & Galena & +10.8 & Near portal \\
\hline
\end{tabular}

\footnotetext{
analyses by Knueger Enterprizes Inc., Geochron Laboralories Division, Cambridge, Massachuserts. Unless otherwise noted analyses are reported in 0/00 notation and are computed as follows:

$$
\mathrm{S}^{34} \text { sample } 0 / 00=\frac{{ }^{34} \mathrm{~S} \mathrm{H}^{32} \mathrm{~S} \text { sample } \times 1,000}{{ }^{34} \mathrm{~S}^{32} \mathrm{~S} \text { standard }}
$$

bAlso could be stared as "del-34 value."
} 


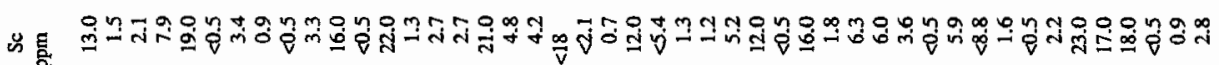

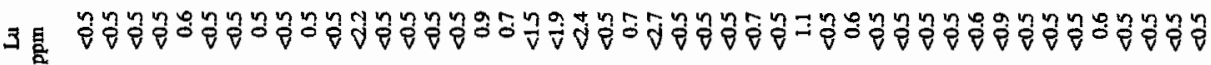

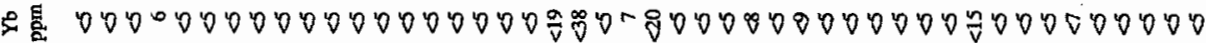

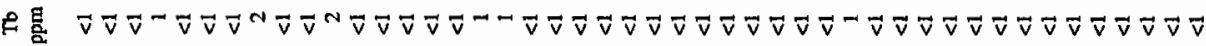

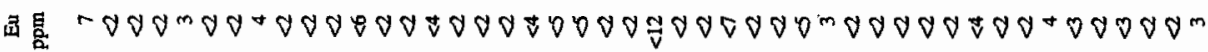

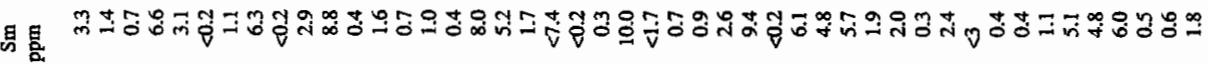

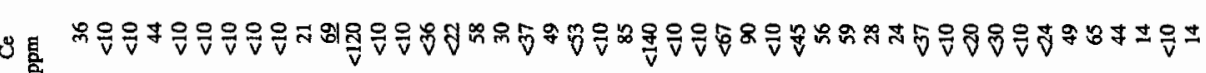

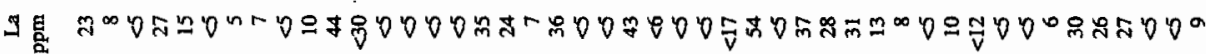

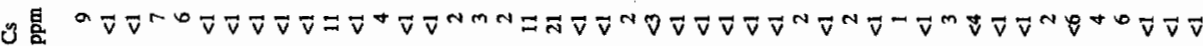

อ 里毠

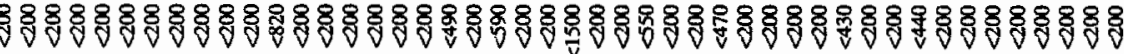
量

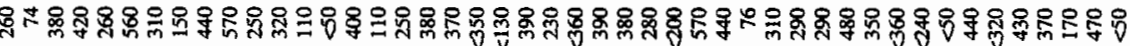

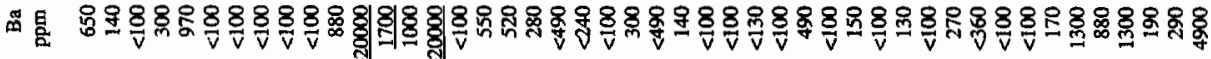

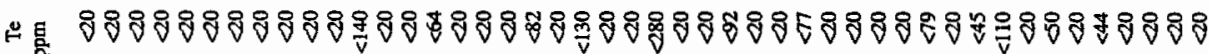

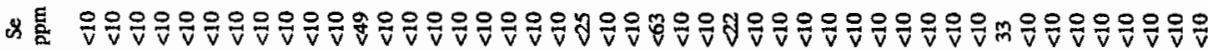

出 ถ

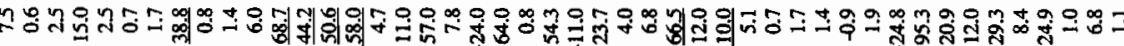

子喜

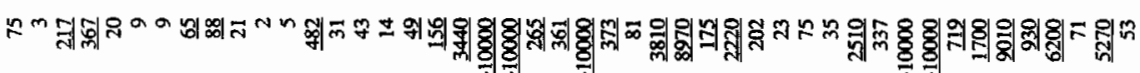

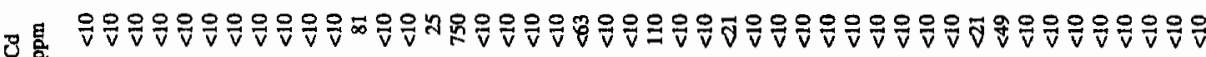

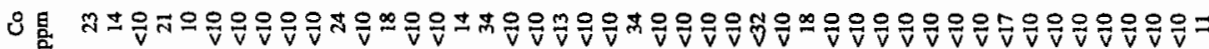

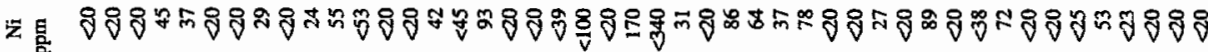

自

禾

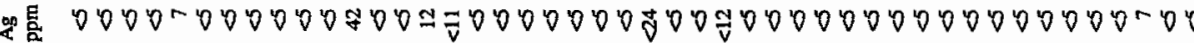

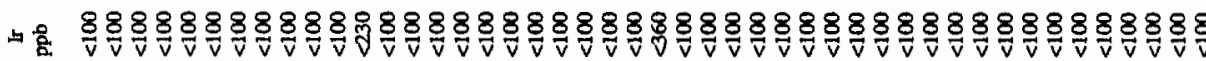
灵京

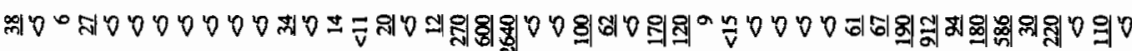

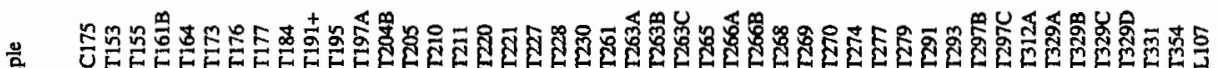

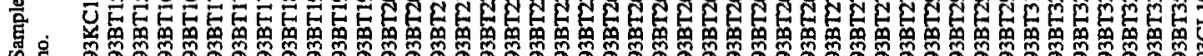




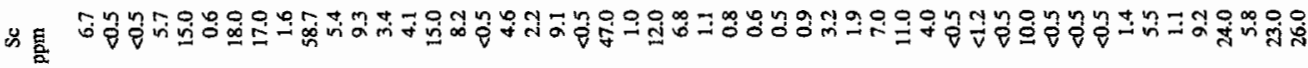

3

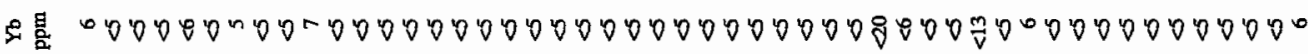

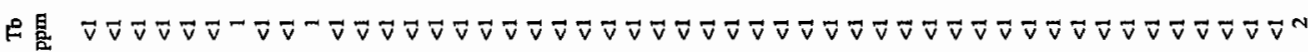

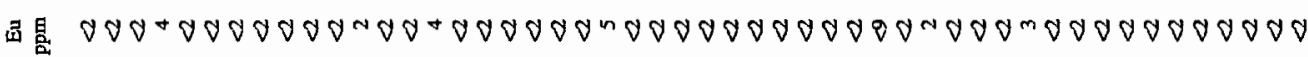

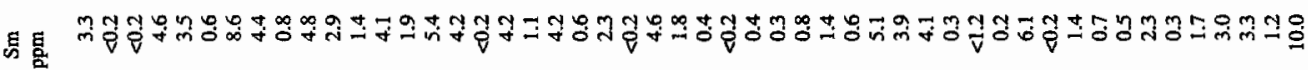

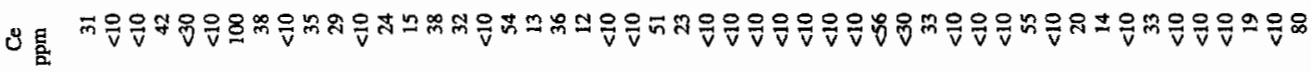

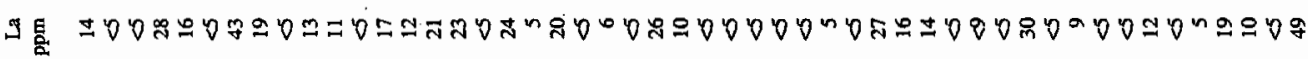

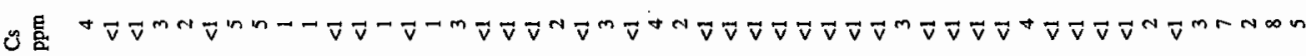

3

क

○最

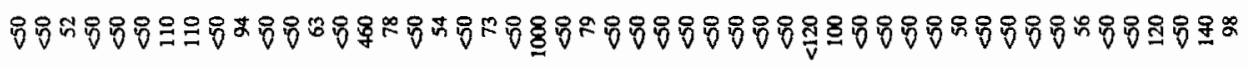

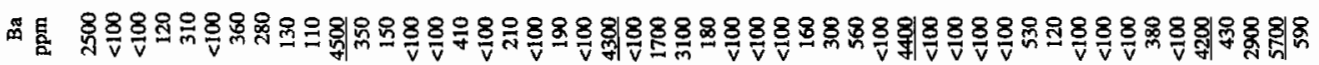

แ

か

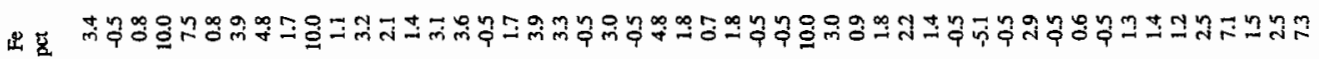

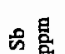

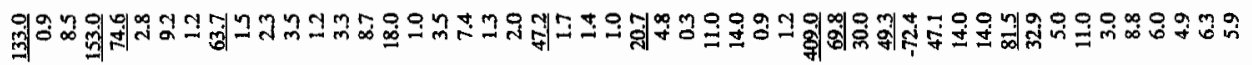

曼

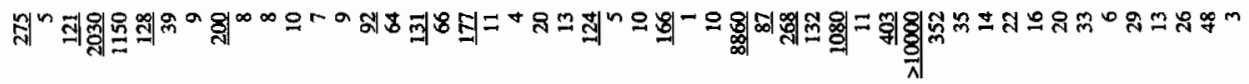

孔

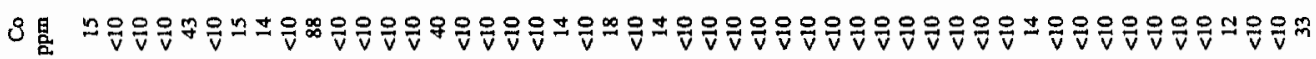

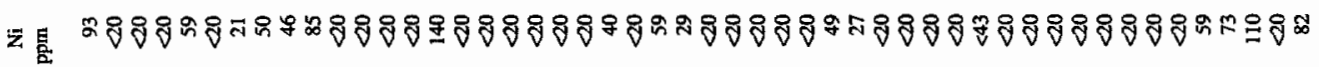

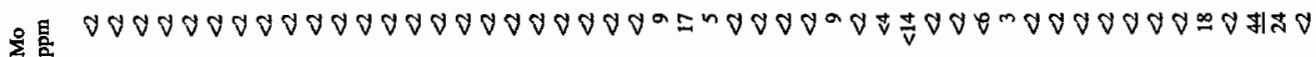

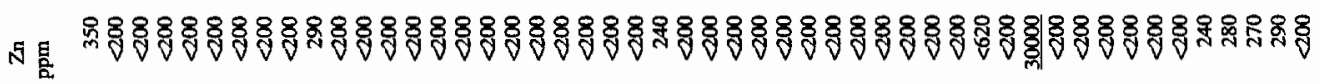

丝 00000000000000000000000000000000000900000000000000

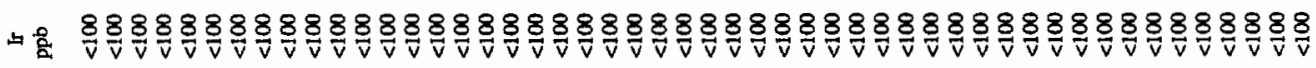

至ํㅠㄹ

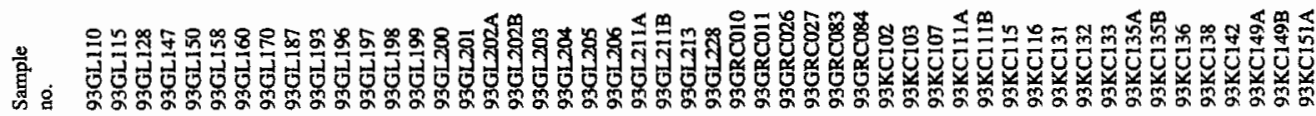




\begin{tabular}{|c|c|c|c|c|c|c|c|c|c|c|c|c|c|c|c|c|c|c|c|c|c|c|c|c|c|c|}
\hline $\begin{array}{l}\text { Simpie } \\
\text { nsm }\end{array}$ & $\begin{array}{l}\mathrm{Aur} \\
\mathrm{Ppb}\end{array}$ & $\underset{\substack{\text { If } \\
\mathrm{ppb}}}{ }$ & $\begin{array}{l}\mathrm{Ag} \\
\mathrm{Pp}\end{array}$ & $\underset{\text { patin }}{\text { Zn }}$ & $\begin{array}{l}\text { Mo } \\
\text { PQDO }\end{array}$ & $\underset{p p m}{N}$ & $\underset{m p}{6}$ & $\underset{\mathrm{cppm}}{\mathrm{cd}}$ & As & $\begin{array}{l}5 b \\
\mathrm{ppmit}\end{array}$ & $\underset{f t}{E x t}$ & $\underset{\mathrm{pet}}{\mathrm{se}}$ & $\begin{array}{l}T_{e} \\
\text { ppln }\end{array}$ & Ppan & $\stackrel{\mathrm{O}}{\mathrm{pppm}}$ & 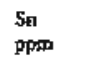 & $\begin{array}{l}\text { w } \\
\text { ppint }\end{array}$ & $\mathrm{cps}_{\mathrm{ppo}}$ & $\underset{\text { L }}{\mathrm{L}}$ & $\underset{\mathrm{ppg}}{\mathrm{C}}$ & $\begin{array}{r}\text { Sm } \\
\text { pplum }\end{array}$ & $\begin{array}{r}\text { Eئ } \\
\text { PpI }\end{array}$ & $\begin{array}{r}\text { Tb } \\
\text { pput }\end{array}$ & $\begin{array}{c}\mathrm{Yb} \\
\text { ppmil }\end{array}$ & $\begin{array}{r}\text { Lu } \\
\text { ppon }\end{array}$ & $\begin{array}{r}\mathbf{s c} \\
\text { ppor }\end{array}$ \\
\hline $93 \mathrm{KCLSIB}$ & 7 & $\epsilon+00$ & $\sigma$ & 210 & $a$ & $\infty 0$ & $<10$ & $\in F 0$ & 1] & 2.5 & 5.6 & $<10$ & $\infty 0$ & 510 & 170 & 200 & $\infty$ & 11 & 15 & 23 & 1.7 & $a$ & 4 & $\sigma$ & 0.9 & 27.0 \\
\hline $93 \mathrm{KCl} 63$ & $\sigma$ & $<100$ & क & 280 & 8 & 64 & 68 & $<10$ & 72 & 140 & 100.0 & $=10$ & 20 & 200 & 110 & 2000 & 8 & 2 & 9 & $<10$ & 32 & 2 & $<\mathrm{l}$ & 6 & 0.7 & 26.0 \\
\hline $93 \mathrm{KCl} 170$ & 23 & $<100$ & 0 & 200 & 0 & 20 & $<10$ & $<10$ & 1219 & 200 & 1.1 & $<30$ & 246 & 260 & so & $\$ 00$ & 6 & 9 & 70 & 110 & R.3 & 5 & $<1$ & 4 & 0.9 & 11.0 \\
\hline $93 \mathrm{KC171}$ & $\delta$ & $\angle 100$ & 10 & 80 & 8 & 20 & 410 & $\leqslant 10$ & 72 & 55 & 09 & $=10$ & 80 & 210 & 91 & 000 & 10 & 28 & 69 & 120 & 7.6 & 2 & 2 & 6 & 0.7 & 8.7 \\
\hline $93 \mathrm{KCI} \pi$ & 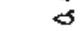 & $=100$ & d & 200 & 0 & $\infty 0$ & $\approx 10$ & $\Leftrightarrow D$ & 6 & 22 & -0.5 & $<10$ & 20 & 400 & $\infty 0$ & 200 & $\approx 2$ & $<1$ & 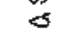 & $<10$ & 0.3 & 2 & $<i$ & 8 & dos & 0.7 \\
\hline $93 \mathrm{KCI}$ S5A & $\delta$ & $<100$ & 5 & 200 & 2 & 20 & $<10$ & 810 & 6 & 1.9 & 1.2 & $<10$ & 20 & $<100$ & $\infty$ & 000 & 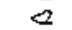 & $\Leftrightarrow$ & 18 & 29 & 3.0 & 0 & $<i$ & 5 & 0.5 & 35 \\
\hline $93 \mathrm{KC1958}$ & 19 & $<100$ & 0 & 800 & 8 & $\infty$ & 810 & $<10$ & 15 & 200 & 3.8 & $<0$ & 20 & $<100$ & 80 & 2000 & $a$ & $\Leftrightarrow$ & 40 & 79 & 6.3 & 3 & $\Leftrightarrow \mathrm{t}$ & 5 & 005 & 6.0 \\
\hline $93 \mathrm{KCO} 02$ & 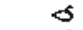 & $=100$ & 8 & 200 & $<$ & 20 & $<10$ & $<10$ & 3 & 1.9 & -0.5 & 470 & 20 & 110 & 50 & 200 & 2 & $<1$ & 6 & jo & 0.9 & 2 & $\&$ & $\sigma$ & 80.5 & 1.0 \\
\hline 93KCOIA & $\sigma$ & $=100$ & 5 & 220 & 2 & 85 & 33 & $<10$ & 3 & 4.0 & 6.2 & $<10$ & 0 & 620 & 140 & $\infty \infty 0$ & 2 & 5 & 40 & 81 & 6.9 & 2 & $<1$ & $\delta$ & 0,7 & 24.0 \\
\hline $93 \mathrm{KCZ} 15$ & $\sigma$ & $\approx 100$ & of & $\infty 00$ & 0 & 20 & 23 & $<10$ & 2 & 3.9 & 4.8 & दा० & 20 & slo & 100 & 2000 & 2 & 4 & 45 & 75 & 7.8 & 5 & \&] & 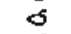 & 0.6 & 18.0 \\
\hline $93 \mathrm{KCOLI}$ & 5 & द] 100 & $\Phi$ & 000 & 2 & 20 & $<10$ & $<10$ & 17 & 21 & 0.6 & $<10$ & $\infty 0$ & 110 & 50 & 200 & 2 & <1 & $\delta$ & 200 & 0.7 & 8 & $<1$ & 5 & 50.5 & 23 \\
\hline $93 \mathrm{KCON}$ & 19 & $\varangle 100$ & 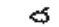 & 800 & 0 & 32 & $<10$ & Q10 & 261 & 78.6 & 26 & <10 & 20 & 170 & $\$ 0$ & 200 & 2 & $<\mathrm{l}$ & $\delta$ & $<10$ & 0.4 & 8 & \&ा & 5 & $\infty .5$ & 3.4 \\
\hline 935104 & 8 & 800 & 6 & 200 & $\ddot{a}$ & $\infty 0$ & $<10$ & alo & 38 & 1.4 & -0.5 & $<10$ & 80 & 400 & $\infty$ & $<00$ & $a$ & \&। & 5 & $\leqslant 10$ & 0.2 & $d$ & $<$ & 5 & $\$ 3$ & 80 \\
\hline 91SL2>D & 5 & $<100$ & $b$ & 9400 & 2 & $\infty 0$ & $<10$ & $\mathfrak{3}$ & 110 & 4in & 1.4 & $<10$ & 80 & $<100$ & $\infty_{0}$ & 800 & 3 & $<1$ & 11 & $<10$ & 110 & 7 & 3 & 7 & 0.8 & 1.4 \\
\hline 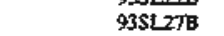 & $z$ & $<100$ & 8 & 200 & 3 & 59 & 14 & $<10$ & 1490 & $\frac{1107}{140}$ & 5.0 & $<0$ & 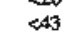 & 410 & $\infty 0$ & 200 & 8 & 1 & 8 & 21 & 1.8 & $i$ & أه & $\dot{0}$ & 0.5 & 1.9 \\
\hline 925125 & $\$$ & 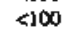 & $\delta$ & 200 & 4 & 20 & $<10$ & $<10$ & 19 & 0.9 & 5.8 & 40 & 80 & $<100$ & 00 & 200 & 8 & $<t$ & 5 & 12 & 3.5 & 3 & 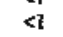 & 8 & $\infty .5$ & 0.7 \\
\hline 935100 & $\delta$ & 40 & 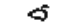 & 200 & 0 & 80 & $<10$ & alo & 2 & 28 & 0.5 & 40 & $\infty 0$ & $=100$ & $\infty$ & 200 & 2 & $<1$ & $\phi$ & 410 & 0.2 & 2 & $<\mathrm{t}$ & $\delta$ & $\$ .5$ & $\infty .5$ \\
\hline 93SE47A & 26 & $<100$ & 4 & 200 & 2 & $\infty 0$ & $<10$ & $<10$ & 62 & 29.8 & 1.2 & sjo & $\infty 0$ & 100 & $\infty$ & $<00$ & 2 & $<1$ & $\sigma$ & $<10$ & 05 & $\bar{a}$ & $<1$ & 5 & 0.5 & 1.1 \\
\hline $933 L, 4 \pi$ & $\delta$ & $<100$ & 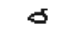 & $\infty 00$ & 0 & 40 & 24 & $\leq 10$ & 25 & 3.2 & 4.6. & $<10$ & 20 & 160 & 110 & 000 & $\bar{c}$ & 1 & 49 & 85 & 9.0 & 4 & 1 & 8 & 0.5 & 15.0 \\
\hline 93SLA7C & 5 & $<100$ & 5 & 800 & 8 & 20 & 810 & $<10$ & 38 & 5.1 & 1.3 & $<10$ & 80 & $<100$ & 53 & 800 & 2 & \& 1 & 7 & elo & 1.5 & 2 & $\mathrm{cl}$ & 5 & $\leqslant 0.5$ & 8.3 \\
\hline 93SL499 & 6 & $<100$ & 0 & 350 & 8 & 40 & 70 & $<10$ & 5 & is & 100 & $<10$ & $\infty 0$ & 8100 & 180 & 8000 & 8 & 1 & $j$ & 40 & 4.8 & 2 & $\mathrm{j}$ & 7 & 0.9 & 548 \\
\hline gasLSo & 14 & $<100$ & 5 & 2500 & 2 & 94 & 28 & $<10$ & 553 & 483 & 10.0 & $<10$ & 80 & 140 & $\infty$ & $\infty 00$ & 2 & 7 & 11 & 23 & 2.2 & 2 & 4 & S & ols & 11.0 \\
\hline 935I_SIA & 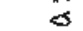 & $<100$ & $\varepsilon$ & 000 & 2 & $\infty$ & $<10$ & $\leq 10$ & 6 & 1.7 & 0.9 & $<10$ & 80 & $<100$ & $\infty$ & 200 & $\Leftrightarrow$ & 4 & 8 & $\leqslant 10$ & 1.2 & 2 & 4 & 8 & $\infty$ & is \\
\hline YSSL $\leqslant 1$ & $\delta$ & $<100$ & s & 200 & 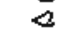 & $\infty 0$ & $<10$ & $<0$ & 2 & 0.6 & -0.5 & 90 & 20 & 130 & $\infty 0$ & 200 & 2 & $i$ & 7 & 13 & 1.4 & 2 & $a$ & 8 & $\$ 5$ & 12 \\
\hline 93SISTA & 5 & $<100$ & $\varnothing$ & 200 & 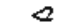 & 20 & $<10$ & $<10$ & 2 & 0.6 & 0.9 & $<10$ & $\infty$ & 100 & $\infty$ & 200 & 2 & $<1$ & $\delta$ & $<10$ & 0.9 & $a$ & $<1$ & $\sigma$ & $\infty .5$ & 3.t \\
\hline 93SL62A & $\delta$ & $<100$ & 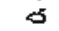 & 000 & $\Leftrightarrow$ & 33 & 810 & 40 & -1 & 0.5 & 3.4 & $=10$ & $\infty$ & 520 & $\$ 0$ & $\infty 00$ & 8 & 3 & 4 & 83 & 78 & 5 & 1 & $\delta$ & 0.7 & 120 \\
\hline $935 \mathrm{LSgA}$ & o & $<100$ & $\delta$ & 200 & $a$ & $\infty$ & $<10$ & <10 & 27 & 263 & 22 & & $\infty 0$ & 310 & $\infty$ & 200 & 2 & I & 8 & $<10$ & 15 & 2 & i & $\sigma$ & 0.5 & 2.2 \\
\hline gsit. 75 & 5 & $<100$ & 5 & 280 & $a$ & 120 & 74 & $<10$ & 5 & 6.5 & 100 & $<0$ & 20 & 110 & 40 & 200 & 3 & i & 10 & 25 & 3.8 & 4 & $<1$ & $\delta$ & 0.8 & 60.4 \\
\hline 9352764 & 6 & $<100$ & 6 & 640 & 4 & 80 & $<0$ & $<10$ & $15 x$ & 19,0 & 30.0 & $<10$ & $\infty$ & 360 & 80 & $\infty \infty$ & $a$ & $<1$ & 8 & $<10$ & t.1 & 2 & $<1$ & 6 & 60.5 & $\$ 3$ \\
\hline 935L.766 & 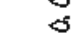 & $\leqslant 100$ & 6 & 800 & 2 & $\approx 0$ & clo & $\leqslant 10$ & $\frac{1}{\mathbf{z}}$ & 27.4 & 3.6 & $<10$ & 20 & 120 & 50 & 200 & $a$ & $\leqslant 1$ & 8 & $<10$ & 0.6 & 2 & $<1$ & 8 & 80.5 & 8.5 \\
\hline 935L78B & 8 & $<100$ & $\infty$ & 200 & 0 & $\infty$ & $<10$ & $\Leftrightarrow$ & $\frac{\alpha 15}{57}$ & 27 & 10.0 & $<10$ & 00 & 400 & 50 & $\infty \infty$ & 8 & 2 & 8 & $<10$ & 0.6 & 0 & $\leqslant 1$ & है & 60.5 & 2.7 \\
\hline $93517 \% C$ & $d$ & $<100$ & $d$ & 240 & 2 & 78 & 75 & \&l & $\frac{1}{3}$ & 1.8 & 10.0 & 810 & 20 & $<100$ & 350 & 200 & 2 & 3 & 10 & Zz & 3.7 & 3 & $\& 1$ & -5 & 0.7 & 569 \\
\hline 93SLIZA & $d$ & $<100$ & 8 & 200 & 0 & $\infty$ & $<10$ & $<10$ & 8 & 39 & 13 & $\approx 10$ & 20 & 250 & 80 & 200 & 2 & $\measuredangle t$ & 6 & $<10$ & 1.1 & 2 & \& & $\sigma$ & $\operatorname{sos}$ & 3 \\
\hline 99SL 99 & 5 & $<100$ & 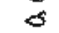 & 200 & 2 & 20 & $=10$ & $<10$ & 2 & 0.6 & $i .7$ & 40 & 20 & 220 & $\Leftrightarrow$ & $\infty$ & $\varepsilon$ & 2 & $\delta$ & $<0$ & J.I & 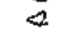 & $4 !$ & 5 & & 5.6 \\
\hline 935L:1068 & $\delta$ & $=100$ & $\delta$ & $\$ 00$ & $a$ & $\infty$ & $\leq 10$ & $<10$ & 1 & 0.5 & 5.6 & 40 & $\infty 0$ & 400 & so & 200 & 2 & $<1$ & 11 & 16 & 5.1 & $\Leftrightarrow$ & $<\mathrm{l}$ & 5 & $\$ 0.5$ & 4.2 \\
\hline 93SL [ 178 & 21 & $\rightarrow 100$ & 5 & 200 & 2 & 80 & 810 & $<10$ & 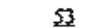 & 573 & 1.0 & $<10$ & 80 & $<100$ & so & 2000 & 0 & $<1$ & 5 & $<10$ & 2.1 & 2 & $\ll 1$ & $\Leftrightarrow$ & 005 & 0.7 \\
\hline $9351117 \mathrm{C}$ & 7 & 5100 & $\bar{\sigma}$ & 200 & $\bar{a}$ & 20 & \&10 & $<10$ & 335 & 160 & 1.0 & $<10$ & $\infty$ & $<100$ & 79 & 800 & 2 & $<1$ & 7 & $<10$ & 9.2 & $=$ & $\Leftrightarrow 1$ & 8 & 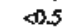 & 1.9 \\
\hline $935 \mathrm{~L} 1209$ & $\delta$ & 8100 & 5 & 200 & 2 & 20 & $<10$ & $<10$ & 5 & 0.7 & 64 & $<10$ & $\infty 0$ & $<100$ & so & 200 & 2 & $<1$ & 5 & $<10$ & 59 & 2 & 2 & 3 & 0.6 & 17.0 \\
\hline 93SL.:23日 & 9 & $<100$ & 5 & 200 & 2 & 36 & 18 & $=10$ & 226 & 5.7 & 100 & $<10$ & $\infty$ & 1800 & 290 & -200 & 3 & 3 & $\delta$ & $<10$ & 1.4 & 2 & a & 5 & 05 & 723 \\
\hline $935 \mathrm{~L} / 42 \mathrm{C}$ & 8 & $<100$ & 5 & 200 & 2 & 2 & 11 & eto & 14 & 73 & 07 & $<10$ & $\infty$ & $\$ 100$ & $\$ 0$ & 2000 & 8 & e & 5 & $<10$ & 0.5 & 2 & 4 & 8 & 0.5 & 21 \\
\hline gisLLISOC & 5 & $<100$ & 5 & 200 & 2 & 36 & II & $<10$ & is & 1.8 & 3.4 & $<10$ & $\infty 0$ & 460 & 50 & 200 & 4 & 1 & 21 & 35 & 4,4 & 2 & $<1$ & क & 0.5 & 6.1 \\
\hline
\end{tabular}


แ

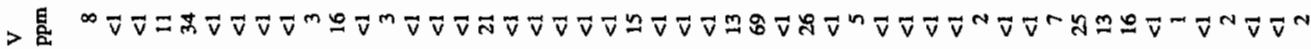

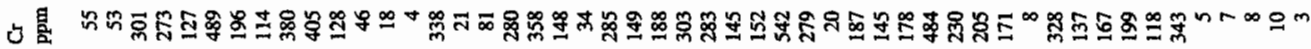

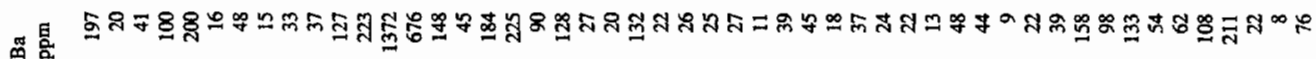

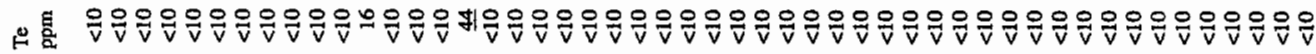

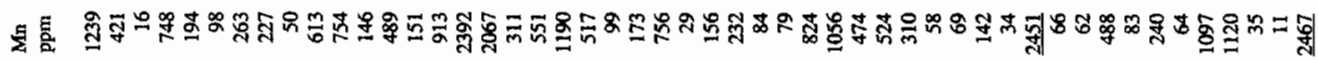

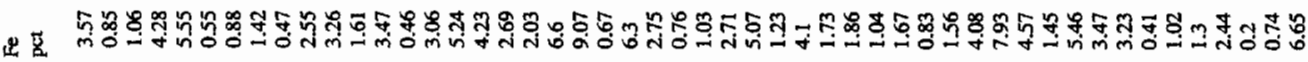

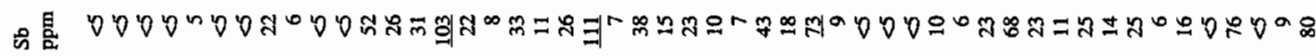
生最

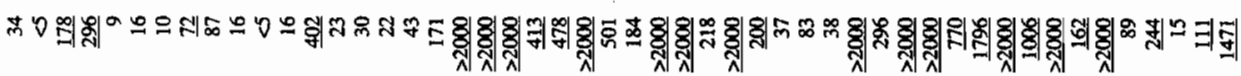

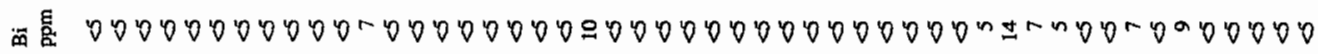
ひ镸

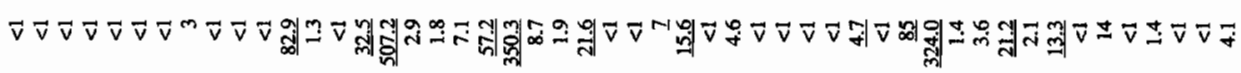

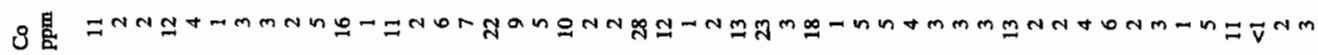
之最

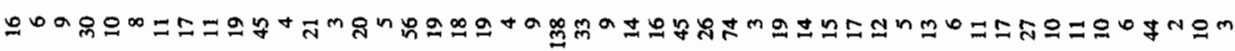
高 ง

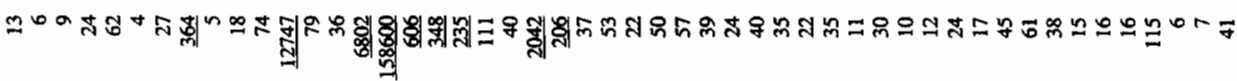
\& 퉁

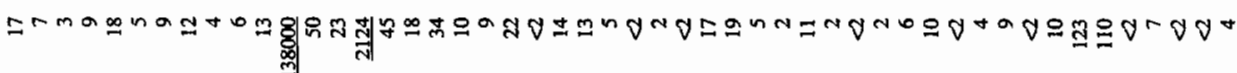
已ี 䂞

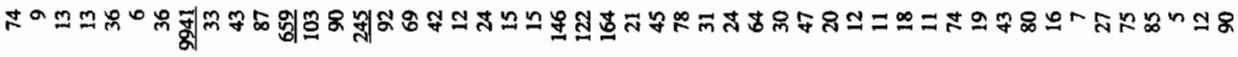
ว 츨 论 电

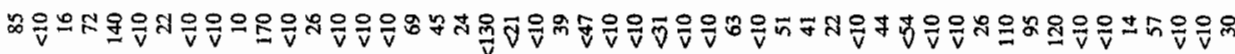
菂星

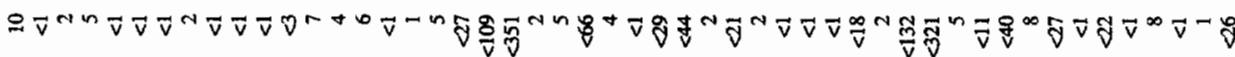

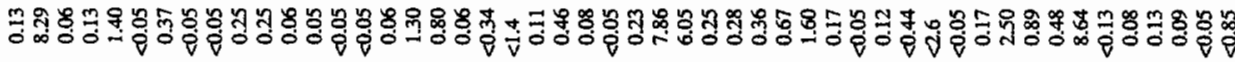
D 镸

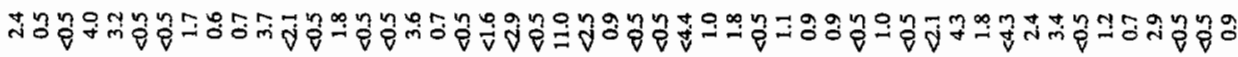
E 莒

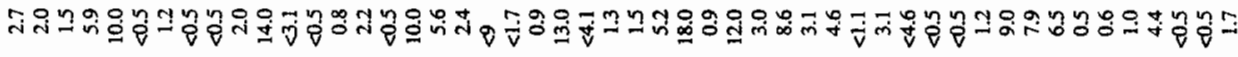

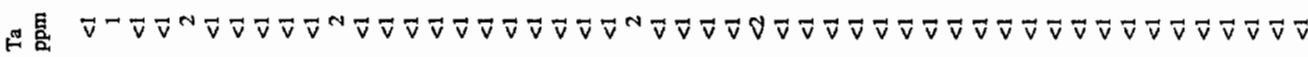
菬

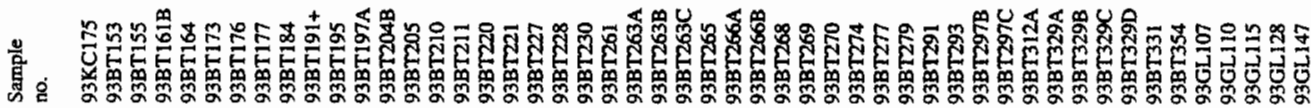


แํㅕㄹ

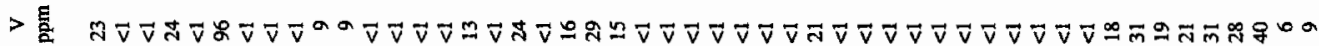

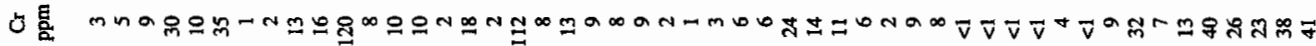

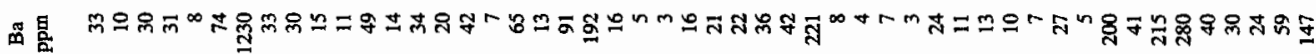

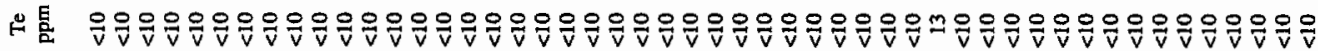

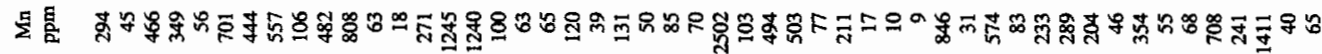

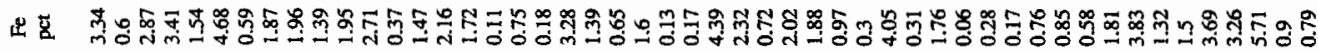

ถิ

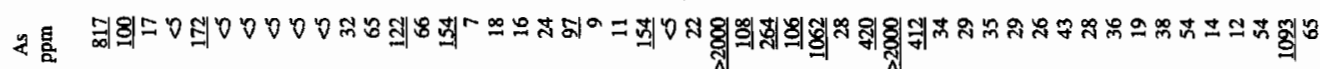

若 00000000000000000000000000000000000000000000000000

品

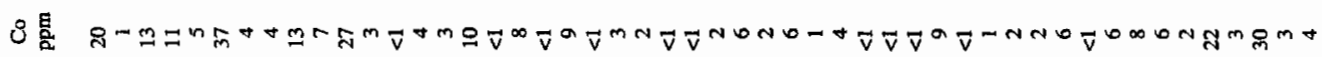

云镸

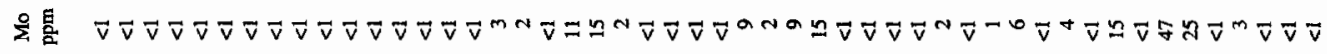
도을

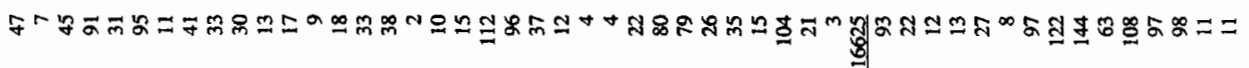
在镸

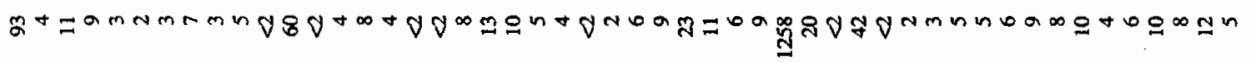

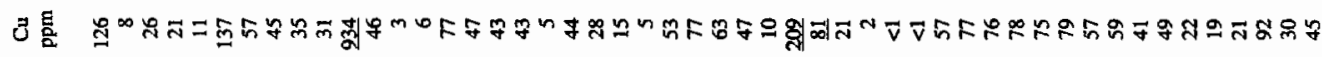

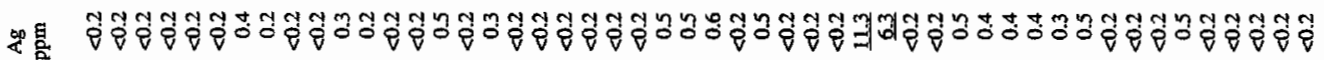

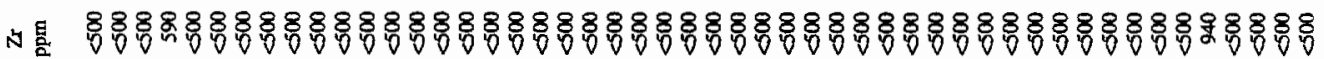
屈

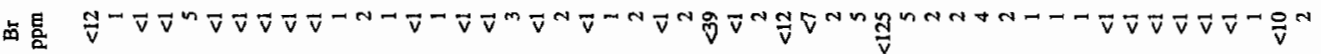

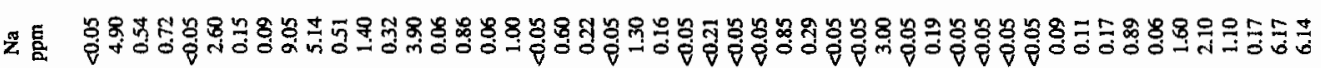

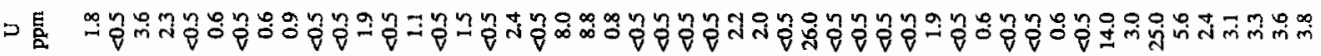
स

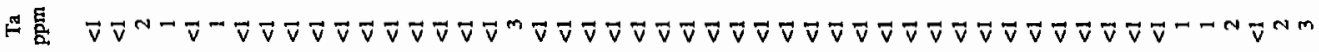

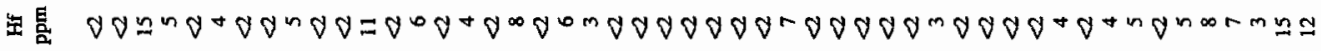

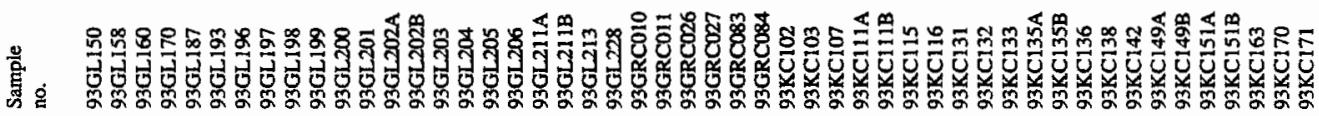




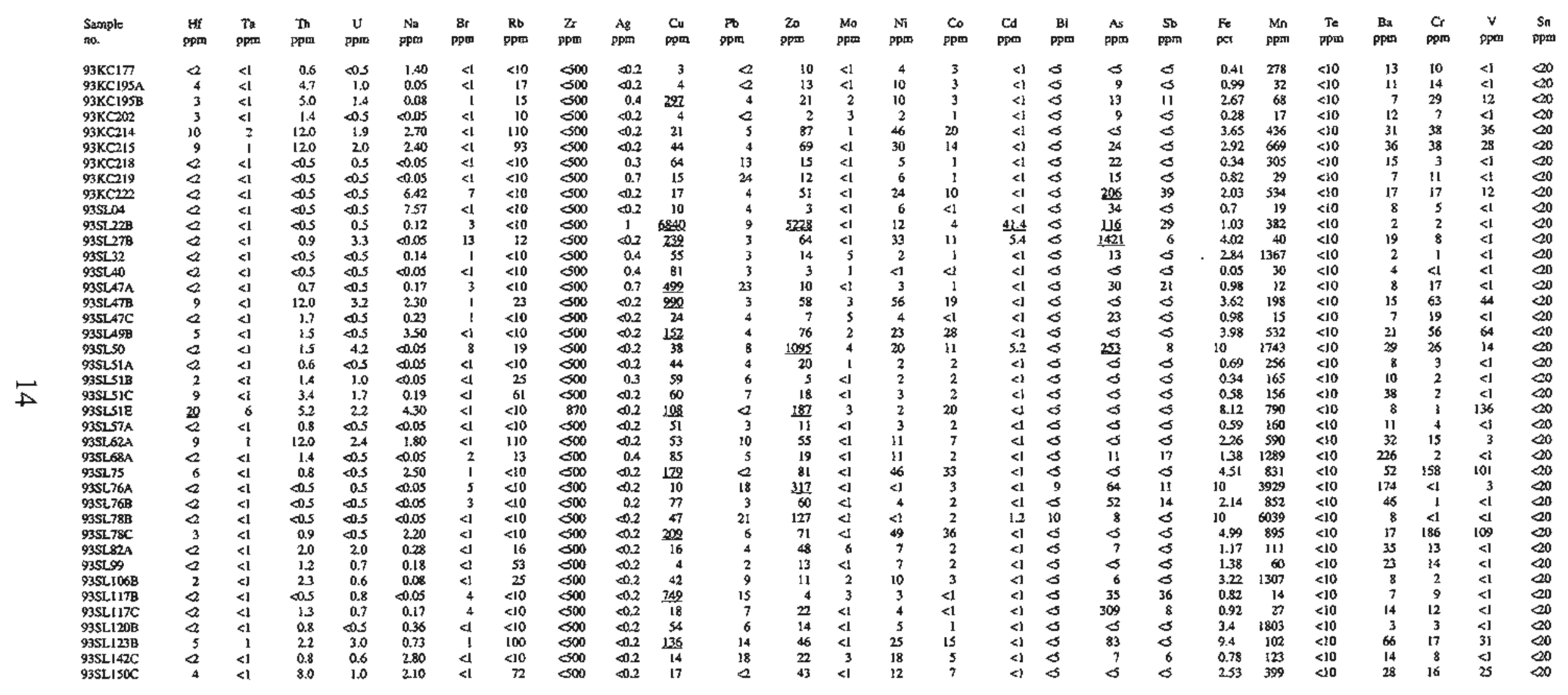




\begin{tabular}{|c|c|c|c|c|c|c|c|c|c|c|c|c|c|c|c|c|c|}
\hline $\begin{array}{l}\text { Sampic } \\
\text { ma. }\end{array}$ & pom & $\begin{array}{c}\text { Lo } \\
\text { ppm }\end{array}$ & A & $\begin{array}{l}\mathrm{Mg} \\
\mathrm{pet}\end{array}$ & $\begin{array}{l}\mathrm{Ca} \\
\mathrm{pad}\end{array}$ & $\begin{array}{c}\text { Ga } \\
\text { ppra }\end{array}$ & $\begin{array}{l}\mathrm{Na} \\
p \mathrm{t}\end{array}$ & $\underset{p=1}{K}$ & $\underset{\text { ppw }}{\mathbf{L i}}$ & $\begin{array}{c}\mathrm{Nb} \\
\mathrm{ppm}\end{array}$ & 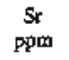 & $\begin{array}{l}\mathbf{T a} \\
\text { gpmim }\end{array}$ & $\begin{array}{c}Y \\
\text { ppw }\end{array}$ & $\begin{array}{l}\mathrm{Ti} \\
\mathrm{pat}\end{array}$ & $\underset{\text { ppm }}{\not \mathbf{r}}$ & $\underset{\mathrm{ppor}}{\mathrm{Su}_{\mathrm{d}}}$ & Description \\
\hline $93 \mathrm{KCl} 5 \mathrm{~s}$ & $\infty 0$ & 10 & 0.71 & 0.22 & 10.00 & 0 & 0.02 & 0.38 & 3 & 38 & 609 & $<100$ & s & $\$ .01$ & $<1$ & 5 & I $\mathrm{m}$ wide zonte, nusty boum soil. \\
\hline $93 \mathrm{BT} 153$ & 20 & 7 & 0.67 & 0.23 & 0.53 & $<$ & 0.36 & 0,02 & 4 & $<1$ & $\frac{20}{43}$ & $<100$ & 4 & $\$ 0.01$ & $<1$ & 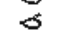 & Quart weio nubble Rodine extration \\
\hline $93 \mathrm{BT} 155$ & $\infty 0$ & 4 & 0.17 & 0.05 & 0.11 & $\dot{b}$ & $<01$ & 0.05 & 2 & 4 & 6 & $<100$ & $i$ & 80.01 & 4 & 13 & 70 est widc, quarte vein, north 5 earl tend; codrtins aspy. \\
\hline $93 \mathrm{BT} 161 \mathrm{~B}$ & 20 & 23 & I.15 & 0.14 & 033 & 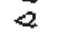 & 0,02 & 0.28 & 6 & 4 & 25 & $<100$ & 12 & $\$ 01$ & $<I$ & 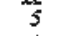 & Gossan in mestublebaic sthis (pCPAn). \\
\hline $93 \mathrm{BT} 164$ & $\infty 0$ & 12 & 3,00 & 1.46 & 0.11 & 12 & 0,07 & 0.59 & 36 & 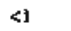 & 15 & $<100$ & 6 & $\$ 01$ & 3 & 9 & Backeround sample, pejitic Solomon sehtist (pCFraph) \\
\hline $938 \mathrm{TITJ}$ & $\infty 0$ & $\Leftrightarrow 1$ & 0.06 & 0.02 & 0.01 & $Q$ & $<0.01$ & $0: 02$ & 2 & $<1$ & 1 & $<100$ & a & a.ol & al & 8 & 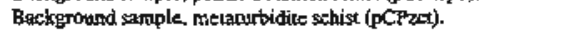 \\
\hline $938 \mathrm{TI} 156$ & $\infty 0$ & 4 & 0.20 & 0.77 & 10.00 & 2 & 0.02 & 0.09 & 2 & 9 & 149 & $<100$ & 5 & 60.01 & $\approx 1$ & 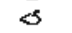 & 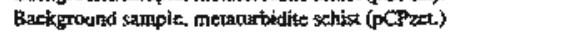 \\
\hline 938 รา 77 & $\$ 0$ & 4 & 0.10 & 0.03 & 1000 & 2 & 0.01 & 004 & 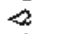 & 9 & 128 & $<100$ & 16 & $\$ 00 t$ & $<1$ & 6 & Chaloopyrite bearing " $F I^{\prime}$ vein; $70 \mathrm{~cm}$ elip chamnet. \\
\hline 938TI84 & 80 & $<t$ & 0.08 & $\infty .01$ & 0.07 & 2 & $=0.01$ & $\infty 01$ & 2 & $\Leftrightarrow$ & 2 & $<100$ & $<$ & 0.04 & \& & 7 & 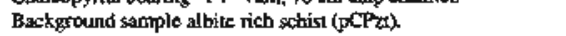 \\
\hline $93 \mathrm{BT} 19 \mathrm{l}+$ & 00 & 8 & 0.59 & 0.45 & 0.18 & 2 & 0,02 & 004 & 10 & a & 7 & $<100$ & 7 & $\$ 0.01$ & \&l & 6 & \\
\hline $938 T 195$ & 20 & $\approx$ & 1.91 & 1.23 & 289 & 3 & 005 & 0.49 & 35 & 3 & 83 & $=100$ & 14 & 0.01 & I & 7 & Mimerebizox layer $20 \mathrm{~cm}$ tick in pCPaf unic. \\
\hline $93 \mathrm{BT} 197 \mathrm{~A}$ & 166 & 3 & 0.12 & 0.03 & 3.75 & 0 & 0,00 & 0,08 & 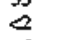 & 4 & 238 & $<100$ & 10 & $=0.01$ & 3 & 8 & 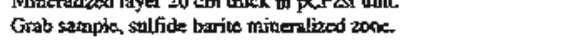 \\
\hline 93872043 & 20 & $<1$ & 0.37 & 020 & 1000 & 2 & 00.01 & 0.11 & 6 & 3 & 42 & 600 & 8 & 80.01 & a & 0 & Minteralized horizon wits uff in $p(P \geq m$ unic \\
\hline $930 \mathrm{~T} 205$ & 20 & \& & 0.14 & 0.06 & 1000 & $a$ & an:ol & 0.06 & $\infty$ & 29 & 260 & $\leqslant 100$ & 4 & 80.01 & el & 0 & 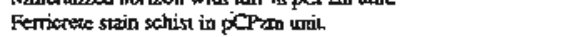 \\
\hline $93 \mathrm{BT} 210$ & Z20 & eा & 1.76 & 0.19 & 230 & 3 & $\infty 01$ & 0.04 & 2 & 3 & 67 & $\leqslant 100$ & 6 & 80.01 & i & 8 & Mineralizod felsic schis (pCPrs). \\
\hline 93ET211 & 进 & <i & 0.04 & 2.28 & 10,00 & 0 & $=0.01$ & 0.02 & 3 & 12 & 42 & $<100$ & 3 & 80.01 & $<1$ & $\delta$ & 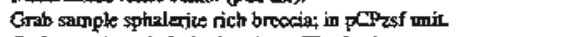 \\
\hline $93 \mathrm{~B} T 220$ & 20 & 8 & 1.93 & 1,14 & $95 \mathrm{t}$ & $\bar{a}$ & 0.05 & 0.19 & 44 & 8 & 96. & $<100$ & 10 & $\$ 0.01$ & $<1$ & $\delta$ & 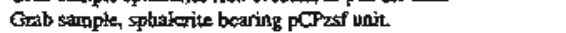 \\
\hline $938 T 221$ & $\infty 0$ & 13 & 0.75 & 0.10 & 3.49 & $\bar{z}$ & 0.05 & 0.4 & $\&$ & 4 & 26 & $<200$ & 9 & 80.01 & 2 & $s$ & Grab simple pQPa schist. \\
\hline $935 \mathrm{ST27}$ & 20 & 7 & 0.44 & 0.05 & 0,56 & 2 & 0.01 & Q.13 & $\Leftrightarrow$ & $<1$ & 13 & $<100$ & 7 & $\$ 0.01$ & 4 & 5 & Twip Wh prospen grab sample. \\
\hline $936 T 228$ & $\infty 0$ & 9 & 0.96 & 0.04 & 0.41 & 8 & 0.02 & 0.34 & 3 & $<1$ & 143 & $<100$ & 24 & $\$ 0.01$ & $<$ & 5 & 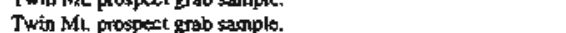 \\
\hline $938 T 230$ & 20 & إع & 0.15 & 0.01 & 0,02 & 10 & 0.03 & 0.02 & 5 & \&i & 6 & $<100$ & \& & 0.01 & $<1$ & 8 & Twin ML prosper gab saople. \\
\hline $95 \mathrm{BT} 2 \mathrm{~K}$ & $\infty$ & 2 & 0.09 & 0.10 & 0.26 & 2 & 0.01 & 001 & $a$ & 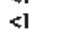 & 4 & $<100$ & \& & 0.01 & $<1$ & 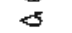 & 2n cm thick $-\mathrm{F}^{2}$ quarte voin in $\mathrm{FCF}$ unit. \\
\hline $93 \mathrm{BT} 263 \mathrm{~A}$ & 20 & 38 & 236 & 0.21 & 0.06 & 8 & 0.02 & 0,28 & 23 & $\Leftrightarrow 1$ & 27 & $=100$ & 14 & $\$ 0.01$ & 4 & 5 & 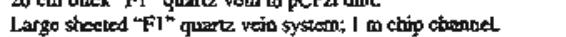 \\
\hline $930 T 263 \mathrm{D}$ & 20 & 5 & 0,3 & 1.08 & 220 & $\alpha$ & 0.01 & 0.02 & 7 & 3 & 16 & $<160$ & 3 & $\$ 0.01$ & \& & 0 & 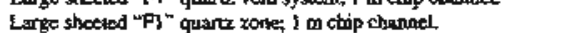 \\
\hline 93В T263C & 20 & 3 & 0.14 & 0.01 & 001 & $a$ & $\infty .01$ & $\cos$ & 2 & द & 2 & $\leqslant 100$ & $\& 1$ & $\infty .01$ & $\Leftrightarrow$ & $\delta$ & 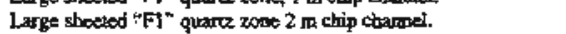 \\
\hline $93 \mathrm{ET} 26 \mathrm{~S}$ & $\infty$ & 4 & 0,15 & 0.04 & 1.72 & $=$ & 0.01 & 0.04 & 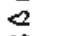 & 2 & 11 & $=100$ & 2 & $\$ 0.01$ & $<1$ & $\Phi$ & N. $40 \mathrm{E}$ urading quartz albice wein. \\
\hline 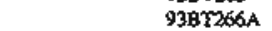 & 20 & 14 & $09 \mathrm{t}$ & 0,51 & 0.05 & 4 & 02 & 0.03 & 12 & $\alpha \bar{t}$ & $\mathrm{~J} A$ & $\$ 100$ & 3 & $\$ 0.01$ & $<1$ & $\sigma$ & Mineralized gossiar near metedionite (Mydg) \\
\hline $93 \mathrm{BT266 \textrm {B }}$ & 20 & 19 & 240 & 1.88 & 0.10 & 15 & 0.08 & $0.0 \mathrm{k}$ & 53 & $\leqslant \mathrm{l}$ & 22 & 900 & 4 & $\$ 0.01$ & 3 & $\delta$ & 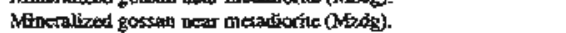 \\
\hline $93 \mathrm{BT2} 288$ & 80 & $\Leftrightarrow$ & 0.17 & 0.03 & 0.30 & $a$ & 0.02 & $\$ 0.01$ & 2 & 4 & 5 & 400 & 4 & $\$ 0.01$ & $\Leftrightarrow$ & 5 & Quart flost zone pearid drodge tilling \\
\hline 9987369 & 20 & 30 & 278 & 1.68 & 025 & 5 & 0.04 & 0.26 & 54 & 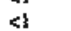 & 19 & $\$ 100$ & II & $\$ 0.0 \mathrm{t}$ & 3 & 9 & Gab sample form Gived portal Rodien propect. \\
\hline $93 \mathrm{BT} 270$ & 20 & 8 & 0.13 & 272 & 1000 & 2 & $\infty .01$ & 0.02 & 4 & $2 \mathrm{I}$ & 382 & $<100$ & 10 & $\infty, 01$ & $<$ & 6 & 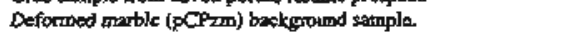 \\
\hline 93BT27A & $\infty$ & 25 & 1.07 & 0.85 & 5.52 & 2 & 0.03 & 0.19 & 11 & 6 & 105 & $<00$ & 9 & $\infty 01$ & 1 & 5 & Quartz-Ibito-gossan veid grab samplo. \\
\hline $938 \mathrm{~mm}$ & 20 & 7 & 0.51 & 0.40 & 10,0 & 2 & 0.03 & 0.12 & 5 & 11 & 190 & $<10$ & 9 & $\$ 01$ & 2 & 8 & 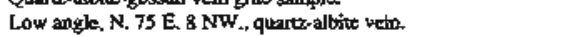 \\
\hline $99 \mathrm{BT} 279$ & 80 & 5 & 0.38 & 0.67 & 2.51 & $a$ & 0.02 & 0.09 & 4 & 3 & 51 & $<100$ & 6 & $=0.01$ & $\mathrm{I}$ & 3 & 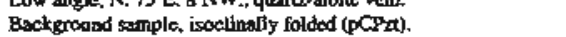 \\
\hline $93 \mathrm{PT}_{2} 1$ & $\infty 0$ & l & 0,04 & 0.02 & 000 & 8 & $\infty 01$ & 0.01 & $\alpha$ & $<1$ & 3 & $<100$ & (1) & $\infty 01$ & $<$ & 5 & 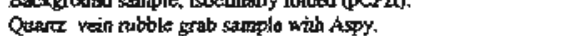 \\
\hline $93 \mathrm{BT} 293$ & 20 & 官 & 0.75 & 0.39 & 0.18 & 3 & 0.02 & 0.15 & 8 & $\leqslant 1$ & 7 & $<190$ & 4 & 001 & 1 & $\$$ & Guarte vetin: $N, 35 \mathrm{~W}$. tion $150 \mathrm{uL}$ \\
\hline $93 \mathrm{BT} 2978$ & 20 & 7 & 0,2 & 0,42 & 009 & 5 & 0.03 & 0.17 & 12 & $<1$ & 12 & $<100$ & 2 & 0001 & $<1$ & 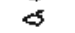 & Aspy-quant vein: 40 cm chip channel, E-W, rend \\
\hline $93 \mathrm{~B} 7297 \mathrm{C}$ & 20 & 4 & 0.10 & 0,02 & $\infty 0 \mathrm{l}$ & 11 & $\$ .01$ & 004 & 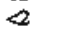 & $<1$ & 2 & $<100$ & آद & 001 & $<1$ & $\$$ & 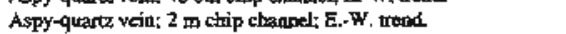 \\
\hline $938 T 3124$ & $\infty$ & $<1$ & 0,04 & 0.63 & 10.00 & 4 & $\Leftrightarrow 0 \mathrm{OJ}$ & 0.01 & 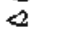 & 24 & 64 & $<100$ & \& & 801 & $\leqslant 1$ & 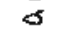 & 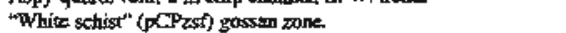 \\
\hline $99 \mathrm{BT} 3 \mathrm{KA}$ & $<0$ & 3 & 020 & 0.08 & 0.21 & 2 & 0.01 & 006 & 2 & $\Leftrightarrow$ & 6 & $<100$ & 1 & $\infty 01$ & 4 & $d$ & Geart wein near old seamp milil: 2 m chip chanroet. \\
\hline $93 \mathrm{BT} 329 \mathrm{~B}$ & 20 & 12 & 0.75 & 0.03 & 0.05 & 8 & 0,06 & 0.3 & 3 & दा & la & $<100$ & 4 & $=0.01$ & 4 & 5 & Qusare vem trear old stamp; $3 \mathrm{~m}$ chtp ctanneli $\mathrm{N} .30 \mathrm{E}$. $55 \mathrm{SE}$ \\
\hline $93 \mathrm{BT} 329 \mathrm{CO}$ & $\infty 0$ & $\mathbf{8}$ & 125 & 0.69 & 1,74 & 4 & 0,05 & 0.22 & 22 & 3 & 0 & $<100$ & 3 & $=0.01$ & 2 & 9 & 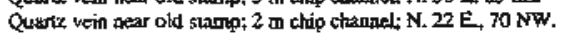 \\
\hline 936T3290 & $\infty 0$ & 10 & 0.78 & 0.26 & 0.69 & 5 & 0.02 & 0.28 & 8 & $\Leftrightarrow$ & 7 & $<1$ & 4 & 0.01 & 4 & $\Leftrightarrow$ & 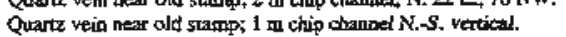 \\
\hline 938T331 & 20 & 2 & 0.26 & $\$ 0.01$ & 0.08 & -2 & 0.2 & $0,0]$ & 2 & दा & 18 & \& & 2 & 000 & $\Leftrightarrow$ & 8 & Grab sample quarz vein. \\
\hline 93BT354 & $\$ \infty$ & 2 & 0.10 & 80.01 & 0.05 & 8 & $<0: 0$ & 0.04 & $\Delta$ & $\Leftrightarrow$ & $2 \$ 7$ & \& & 2 & 00.01 & 1 & 4 & 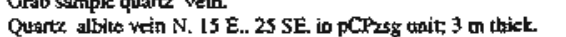 \\
\hline $930 E, 107$ & $\infty$ & 4 & 0.41 & 1.49 & 10.00 & -2 & $\infty, 0\}$ & 0.01 & $<$ & 24 & 340 & $<100$ & 4 & $\infty .01$ & a] & 5 & Sulfides in stringers in calle-sentist. \\
\hline $93 G L 110$ & $\infty$ & 8 & 0.20 & Dof & 0.19 & 2 & $\$ 0.01$ & 0.07 & $\leq$ & $<1$ & 16 & $<100$ & 3 & 0.01 & 2 & $\$$ & 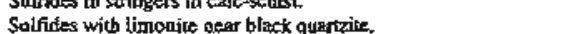 \\
\hline geal Is & $\infty$ & 4 & 0.04 & 0.02 & 0.14 & $\overline{2}$ & $\$ 0,01$ & 00 i & $<$ & 4 & 3 & $<100$ & द & 80.01 & $\Leftrightarrow$ & 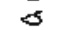 & Quare vein near $\mathrm{pCPa-pCPzet}$ comtar. \\
\hline $90 \mathrm{GL} 128$ & $\infty 0$ & 1 & 0.01 & 000 & 0.02 & $a$ & $\Leftrightarrow 0.01$ & 501 & $\alpha$ & 4 & $\mathrm{t}$ & $<100$ & $=1$ & $\$ 0.01$ & \&1 & 8 & $15 \mathrm{~m}$ thick, quanz v vin. \\
\hline
\end{tabular}




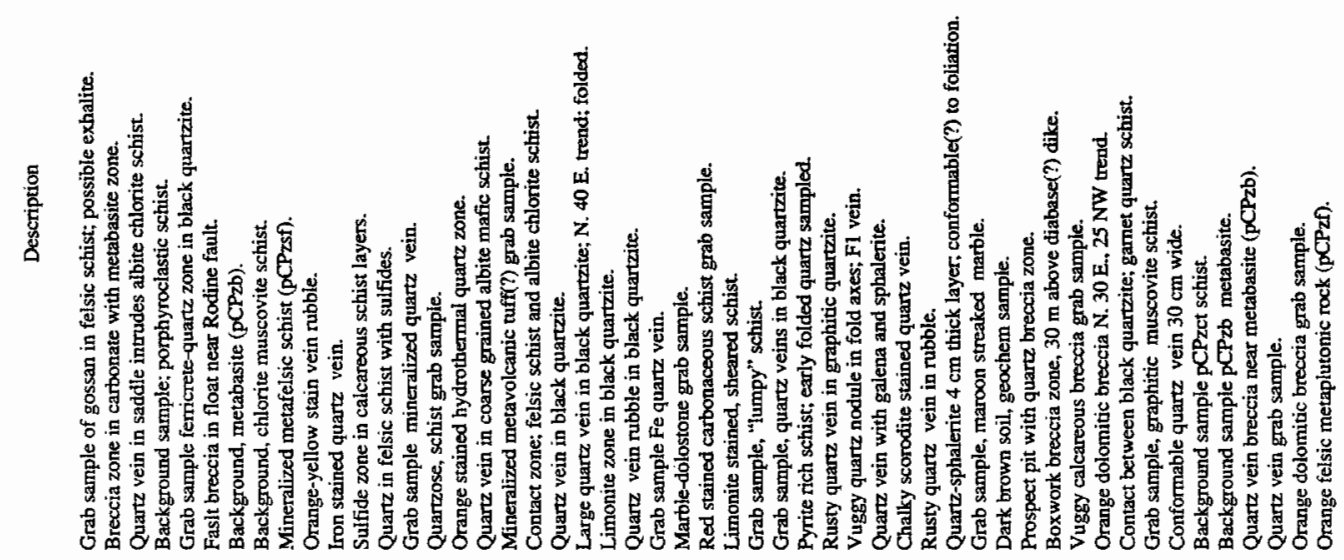

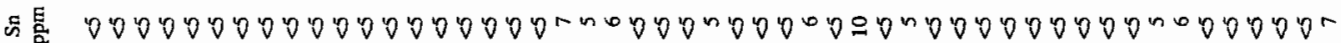

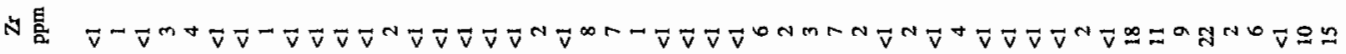

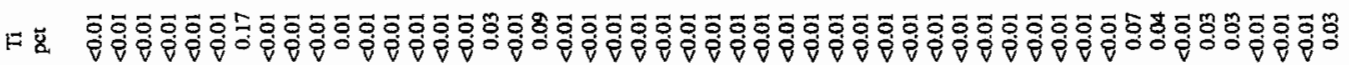

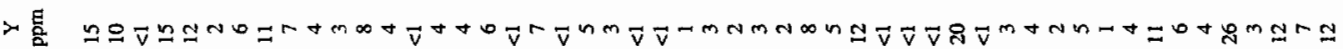

A

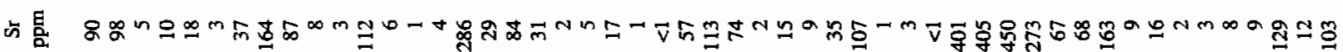

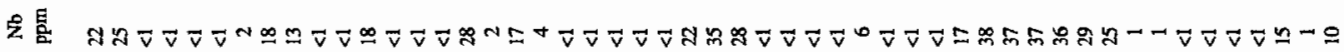

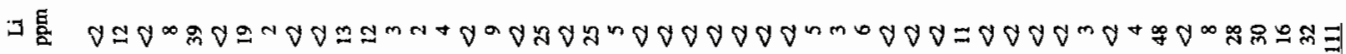

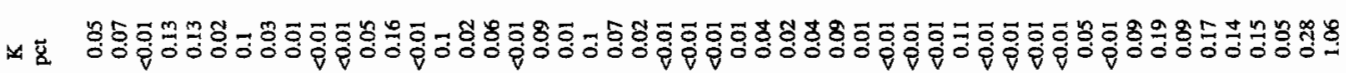

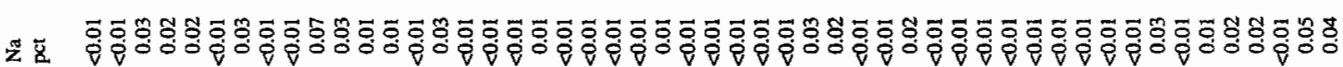

S

ه

之ั

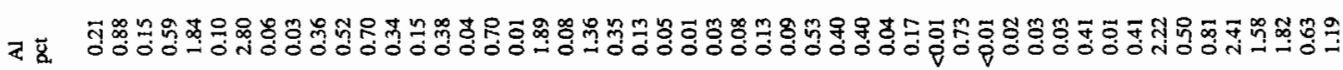

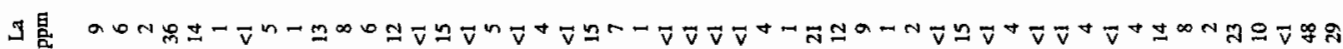

3

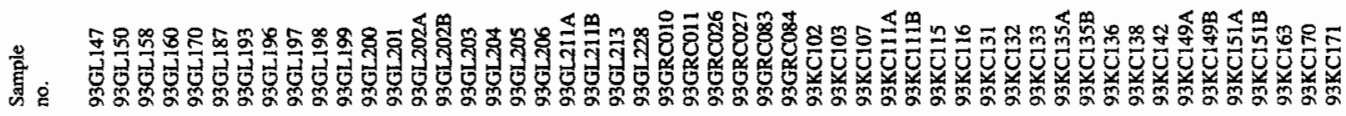




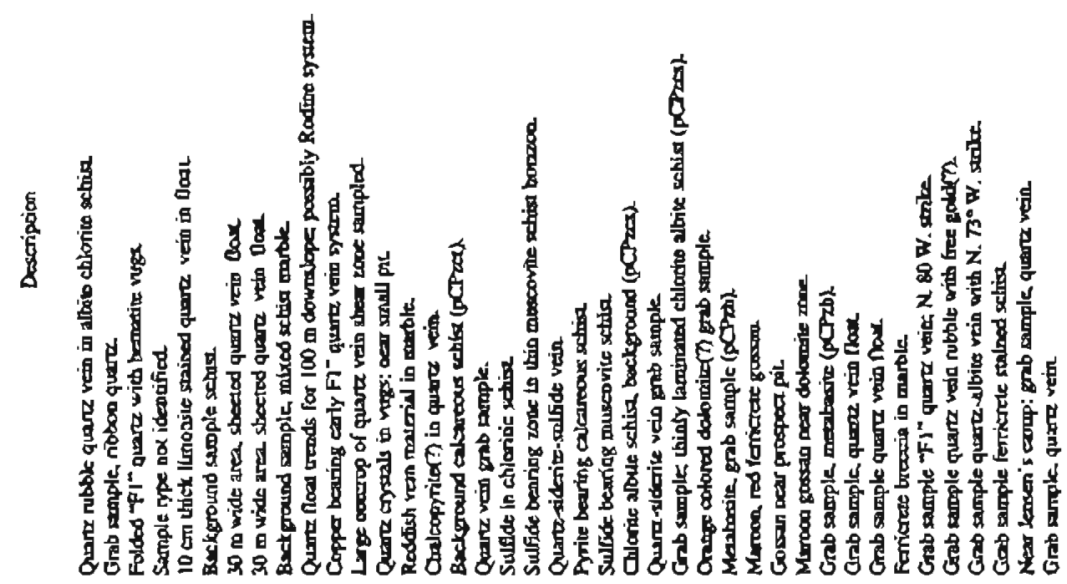

$5 \quad 0000=-000000001000000000000+000000000000$

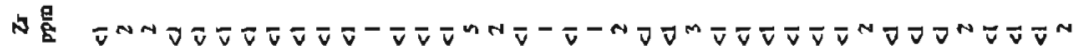

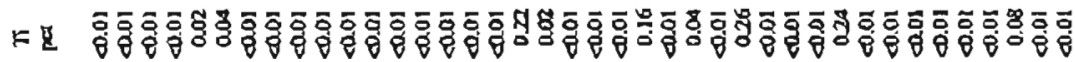

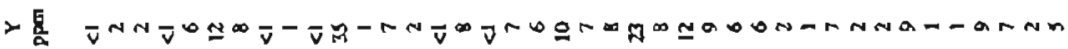

-

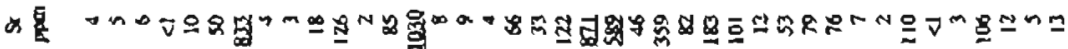

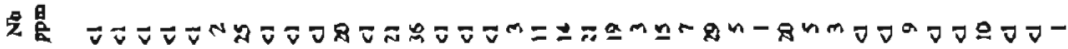

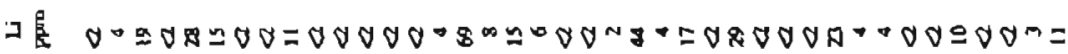

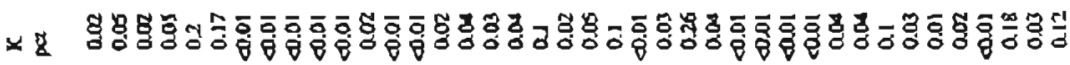

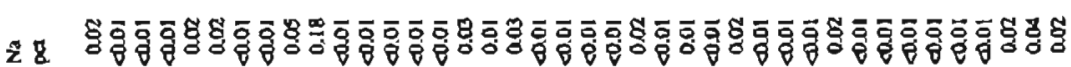

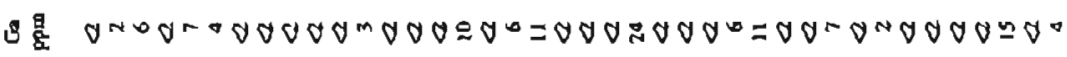

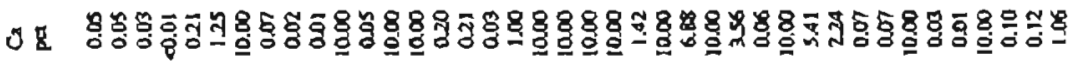

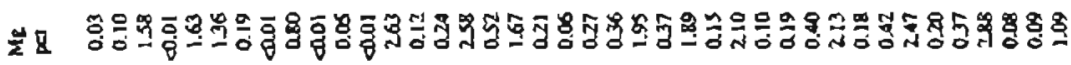

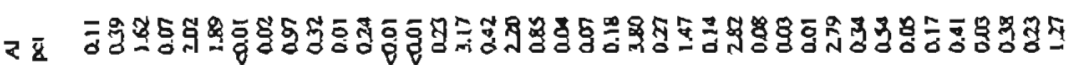

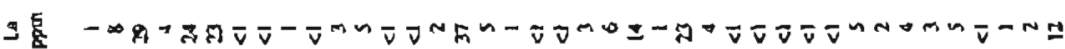

-

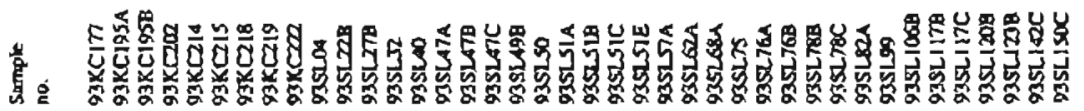


Table 4. Placer gold and silver production, Nome district, $1897.1993^{\circ}$

\begin{tabular}{|c|c|c|c|c|c|}
\hline Year & $\begin{array}{l}\text { Volume gold } \\
\text { (oz) }\end{array}$ & $\begin{array}{l}\text { Vołume silver } \\
\quad \text { (oz) }\end{array}$ & $\begin{array}{l}\text { Number } \\
\text { of mines }\end{array}$ & Employment & $\begin{array}{l}\text { Total bullion valu } \\
\text { ( } \$ \text { at time of sale }\end{array}$ \\
\hline 1897 & 725 & 80 & $\mathrm{NA}$ & $\mathrm{NA}$ & 15,000 \\
\hline 1898 & 3,628 & 409 & NA & NA & 78,000 \\
\hline 1899 & 135,462 & 15,578 & NA & NA & $2,800,000$ \\
\hline 1900 & 166,110 & 20,597 & NA & NA & $3.433,500$ \\
\hline 1901 & 160,619 & 21,041 & NA & $\mathrm{NA}$ & $3,320,000$ \\
\hline 1902 & 152,394 & 17,677 & NA & 3,000 & $3,150,000$ \\
\hline 1903 & 140,299 & 17,537 & NA & 2,800 & $2,900,000$ \\
\hline 1904 & 138,558 & 16,072 & NA & 3,000 & $2,864,000$ \\
\hline 1905 & 164,489 & 20,561 & NA & 3,200 & $3,400,000$ \\
\hline 1906 & 295,114 & 38,659 & NA & 3,500 & $6,100,000$ \\
\hline 1907 & 268,505 & 32,220 & NA & 3,100 & $5,550,000$ \\
\hline $1908-11$ & 664,408 & 81,722 & 135 & NA & $13.730,000$ \\
\hline 1912 & 55,636 & 6,676 & 45 & NA & $1,150,000$ \\
\hline 1913 & 45,800 & 5,038 & 55 & NA & 946,686 \\
\hline 1914 & 120,335 & 14,271 & 65 & NA & $2,487,324$ \\
\hline 1915 & 118,335 & 9,446 & 57 & NA & $2,445,984$ \\
\hline 1916 & 74,600 & 7,460 & 53 & 1,030 & $1,542,000$ \\
\hline 1917 & 42.152 & 5,901 & 40 & NA & 871,281 \\
\hline 1918 & 21,625 & 2,811 & 37 & $\mathrm{NA}$ & 447,000 \\
\hline 1919 & 33,768 & 3.179 & 26 & NA & 698,000 \\
\hline 1920 & 26,124 & 2.775 & 25 & NA & 540,000 \\
\hline $192 I$ & 28,301 & 1.490 & 44 & NA & 585,000 \\
\hline 1922 & 23,463 & 1.121 & 33 & NA & 485,000 \\
\hline 1923 & 28,930 & 3,263 & 21 & NA & 598,000 \\
\hline 1924 & 41.025 & 4,483 & 17 & NA & 848,000 \\
\hline 1925 & 35,800 & 4.244 & 12 & 435 & 740,000 \\
\hline 1926 & 67,247 & 7,389 & 26 & 415 & $1,390,000$ \\
\hline 1927 & 46,105 & 7,137 & 13 & 425 & 953,000 \\
\hline 1928 & 36,284 & 4,094 & 24 & 400 & 750.000 \\
\hline 1929 & 69,762 & 7,299 & 21 & 4500 & $1,442,000$ \\
\hline 1930 & 47,460 & 5,091 & 15 & NA & 981,000 \\
\hline 1931 & 39,157 & 4,850 & 9 & 275 & 809,375 \\
\hline 1932 & 58,129 & 5,742 & 15 & 265 & $1,201,526$ \\
\hline 1933 & 40,912 & 4,541 & 14 & 272 & $845,65 \mathrm{I}$ \\
\hline 1934 & 23,696 & 2,531 & 14 & 250 & 829,360 \\
\hline 1935 & 44,361 & 4,924 & 16 & 340 & $1,551,635$ \\
\hline 1936 & 60,132 & 6,493 & 16 & 325 & $2,104,620$ \\
\hline 1937 & 44,924 & 4,903 & 5 & 345 & $1,572,340$ \\
\hline 1938 & 47,212 & 5,450 & 5 & 343 & $1,652,420$ \\
\hline 1939 & 49,500 & 5,742 & 7 & 365 & $1,739,500$ \\
\hline 1940 & 52,683 & 5,935 & 6 & 377 & $1,843,905$ \\
\hline 1941 & 65,526 & 7,290 & 11 & 345 & $2,293.410$ \\
\hline 1942 & 37,499 & 3.863 & 12 & 340 & $1,312,465$ \\
\hline 1943 & 3,799 & 320 & 4 & 8 & 132,965 \\
\hline 1944 & & & - & - & 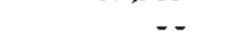 \\
\hline 1945 & 1,193 & 152 & 12 & 16 & 41.755 \\
\hline 1946 & 5,102 & 700 & 24 & 10 & 178,570 \\
\hline 1947 & 5,819 & 610 & 18 & 154 & 203,665 \\
\hline 1948 & 13,576 & 1,590 & 16 & 176 & 475,160 \\
\hline 1949 & 32,302 & 3,202 & 21 & 152 & $1,130,570$ \\
\hline 1950 & 49,418 & 5,498 & 21 & 146 & $1,729,630$ \\
\hline 1951 & 46,465 & 5,189 & 11 & 143 & $1,626,275$ \\
\hline 1952 & 38,318 & 4,345 & 8 & 151 & $1,341,130$ \\
\hline
\end{tabular}

- Compiled by T.K. Bundizen, Alaska Division of Geological \& Geophysical Surveys using (1) U.S. Geological Survey and Terriırial Documenis from 1897-1959; (2) U.S. Mins tecords from 1931-1969; and (3) State of Alaska reconds from 1970-1993. 


\begin{tabular}{|c|c|c|c|c|c|}
\hline 1953 & 29,478 & 3,298 & 15 & 170 & $1,031,730$ \\
\hline 1954 & 21.177 & 2,358 & 15 & 161 & 741,195 \\
\hline 1955 & 23,410 & 2,602 & 14 & 182 & 819,350 \\
\hline 1956 & 24,058 & 2,629 & 8 & 182 & 842,030 \\
\hline 1957 & 43,654 & 4,870 & 7 & 175 & $1,527,890$ \\
\hline 1958 & 33,338 & 3,720 & 7 & 165 & $1,166,830$ \\
\hline 1959 & 873 & 72 & 2 & NA & 30,555 \\
\hline 1960 & 39,050 & 4,443 & 5 & 150 & $1,366,750$ \\
\hline 1961 & 26,442 & 3,076 & 5 & 155 & 925,470 \\
\hline 1962 & 24,001 & 2,727 & 6 & 160 & 840,035 \\
\hline 1963 & 1.272 & 134 & 4 & 3 & 44,520 \\
\hline 1964 & 659 & 74 & 4 & 4 & 23,065 \\
\hline 1965 & 194 & 21 & 4 & 1 & 6,790 \\
\hline 1966 & 41 & 4 & 2 & 1 & 1.435 \\
\hline 1967 & 313 & 14 & 2 & 3 & 3.955 \\
\hline 1968 & 250 & 26 & 1 & 4 & 8.750 \\
\hline 1969.74 & NA & NA & NA & NA & NA \\
\hline 1975 & 7,796 & 1,013 & 1 & 65 & $1,520,220$ \\
\hline 1976 & 14.320 & 1.603 & 3 & 75 & $1,503,600$ \\
\hline 1977 & 15,600 & 2,184 & 3 & 80 & 2.340 .000 \\
\hline 1978 & 12,450 & 1,369 & 3 & 80 & $3,112,500$ \\
\hline 1979 & 14,182 & 1,843 & 4 & 85 & $3,339,600$ \\
\hline 1980 & 12,100 & 1.694 & 3 & 90 & $5,154,600$ \\
\hline 1981 & 16,600 & 1,992 & 4 & 102 & $6,806,000$ \\
\hline 1982 & 18,485 & 2.772 & 4 & 90 & 7.394 .0000 \\
\hline 1983 & 20,500 & 2,542 & 5 & 120 & $8,200,200$ \\
\hline 1984 & 21,500 & 2,601 & 4 & 105 & $7,740,000$ \\
\hline 1985 & 22,416 & 2,689 & 4 & 122 & $7,285,200$ \\
\hline 1986 & 28,650 & 3,151 & 5 & 284 & $10,887,000$ \\
\hline 1987 & 75,824 & 8,340 & 8 & 319 & $34,500,600$ \\
\hline 1988 & 66,500 & 7,700 & 9 & 280 & $28,262,500$ \\
\hline 1989 & 59,500 & 6,950 & 8 & 245 & $22,729,000$ \\
\hline 1990 & 56,300 & 6,193 & 6 & 240 & $21,675,500$ \\
\hline 1991 & 25,700 & 3,048 & 6 & 140 & $9,380,500$ \\
\hline 1992 & 25,600 & 3,584 & 6 & 130 & $8,627,200$ \\
\hline 1993 & 27.750 & 3,607 & 7 & 130 & $9,990,000$ \\
\hline TOTALS & $\begin{array}{l}4,822,569 \\
\text { (149.98 metric } \\
\text { tonnes) }\end{array}$ & $\begin{array}{c}564.134 \\
\text { (17.54 metric } \\
\text { tonnes) }\end{array}$ & - & - & $301,682,072$ \\
\hline
\end{tabular}

